May 28, 2018

\title{
Parafermionic Liouville field theory and instantons on ALE spaces
}

\author{
M. N. Alfimov ${ }^{1,2 *}$ and G. M. Tarnopolsky ${ }^{3 \dagger}$ \\ 1 P.N. Lebedev Physical Institute, Leninskiy pr. 53, 119991 Moscow, Russia \\ 2 Moscow Institute of Physics and Technology, Institutskiy per. 9, 141700 Dolgoprudny, \\ Russia \\ 3 Landau Institute for Theoretical Physics, 142432 Chernogolovka, Russia
}

\begin{abstract}
In this paper we study the correspondence between the $\operatorname{su}(n)_{k} \oplus \operatorname{su}(n)_{p} / \operatorname{su}(n)_{k+p}$ coset conformal field theories and $\mathcal{N}=2 S U(n)$ gauge theories on $\mathbb{R}^{4} / \mathbb{Z}_{p}$. Namely we check the correspondence between the $S U(2)$ Nekrasov partition function on $\mathbb{R}^{4} / \mathbb{Z}_{4}$ and the conformal blocks of the $S_{3}$ parafermion algebra (in $S$ and $D$ modules). We find that they are equal up to the $U(1)$-factor as it was in all cases of AGT-like relations. Studying the structure of the instanton partition function on $\mathbb{R}^{4} / \mathbb{Z}_{p}$ we also find some evidence that this correspondence with arbitrary $p$ takes place up to the $U(1)$-factor.
\end{abstract}

\section{Contents}

\section{Introduction}

2 Instanton counting on $\mathbb{R}^{4} / \mathbb{Z}_{p}$

3 Instanton contributions corresponding to the $S$ and $D$ modules of the $S_{3}$ parafermion algebra

4 Conformal blocks of the $S_{3}$ parafermion algebra

4.1 The extended symmetry algebra of $p=4$ parafermions . . . . . . . . . . . 7

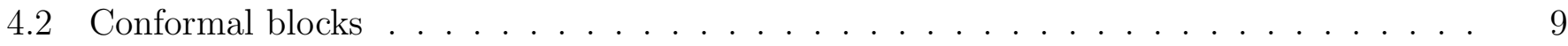

4.2.1 Level $3 / 4$

*E-mail: alfimov-mihail@rambler.ru

${ }^{\dagger}$ E-mail: hetzif@itp.ac.ru 


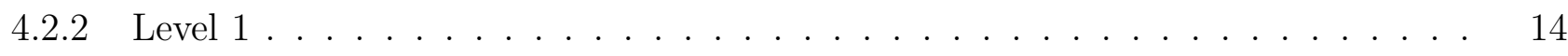

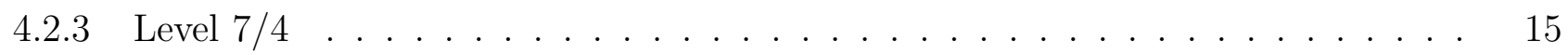

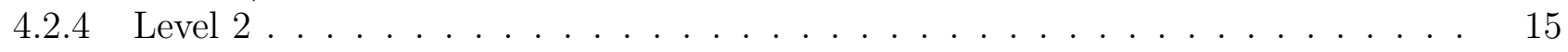

5 Comparing the conformal blocks in the $S$ and $D$ modules of the $S_{3}$ parafermion algebra with the instanton partition function on $\mathbb{R}_{4} / \mathbb{Z}_{p}$

6 Concluding remarks and open problems 17

$\begin{array}{ll}\text { A Commutation relations in the } S \text {-module } & 18\end{array}$

B Commutation relations in the $D$-module 21

C Gram/Shapovalov matrix and matrix elements at the level 7/4 24

D Gram/Shapovalov matrix and matrix elements at the level 2

\section{Introduction}

Two years ago in the seminal paper [1] it was proposed that $\mathcal{N}=2$ supersymmetric $4 \mathrm{~d}$ gauge theories with $S U(2)$ gauge symmetry are related to the 2 d conformal field theory. Since then it has been studied in a lot of papers from the side of gauge theories, conformal field theory [2 6$]$, matrix models [7-10] and abstract math [11,12].

The works [13, 14] proposed the connection between $S U(n)$ gauge theories on $\mathbb{R}^{4} / \mathbb{Z}_{p}$ and coset conformal field theories based on the coset $\hat{\mathrm{su}}(n)_{k} \oplus \operatorname{su}(n)_{p} / \mathrm{su}(n)_{k+p}$, where $p$ is a positive integer and $k$ is a free parameter. The first non-trivial checks for this relation for $p=2$ were made in [15 17] and for $p=4$ in the recent paper by N.Wyllard [18]. The explicit calculations for the case $(n, p)=(2,4)$ were done there in some particular modules and Whittaker limit.

In our paper we proceed with the study of the case $(n, p)=(2,4)$. Namely, we propose the explicit equality between particular Nekrasov instanton partition function on $\mathbb{R}^{4} / \mathbb{Z}_{4}$ and $S_{3}$ parafermion fourpoint conformal blocks in the so called $S$ and $D$ modules [19]. We have checked the correspondence for the first four levels. We show that this equality holds up to the so called $U(1)$-factor, which is the same as in all $S U(2)$ AGT-like relations. It can be schematically represented as

$$
\mathcal{Z}_{\text {instanton }}(z)=(1-z)^{A} \mathcal{F}_{\text {conformal block }}(z),
$$

where $A$ depends on parameters of conformal block (or instanton partition function). Then in the end of the paper we argue, based on explicit evaluation of the instanton partition function on $\mathbb{R}^{4} / \mathbb{Z}_{p}$ for $p=2, \ldots, 7$, that such a relation holds for all $p$. We think that these general relations can be useful for obtaining the answers for the conformal blocks in the parafermion theories using the gauge theory calculations.

The paper is organized as follows. Section 2 is devoted to the instanton counting on the space $\mathbb{R}^{4} / \mathbb{Z}_{p}$. In Section 3 we calculate the Nekrasov partition function on $\mathbb{R}_{4} / \mathbb{Z}_{4}$. In Section 4 we remind some facts about the extended symmetry algebra of $p=4$ parafermions and derive the expressions for the conformal blocks of this algebra. In Section 5 we relate instanton partition function to the parafermion conformal blocks. This relation is the main result of our paper. In Appendices A and B we derive the commutation relations for the vertex operators in $S$ and $D$ modules respectively. In 
Appendices $\mathrm{C}$ and $\mathrm{D}$ we write out the Gram/Shapovalov matrices and matrix elements for the levels $7 / 4$ and 2 respectively.

\section{Instanton counting on $\mathbb{R}^{4} / \mathbb{Z}_{p}$}

In this section we briefly review the counting of the instanton partition function for $\mathcal{N}=2 S U(n)$ gauge theory on $\mathbb{R}^{4} / \mathbb{Z}_{p}[20,21]$. First we describe the case of the pure $\mathcal{N}=2 S U(n)$ gauge theory and then propose more general formulas.

The instanton partition function for the pure $\mathcal{N}=2 S U(n)$ gauge theory on $\mathbb{R}^{4}$ reads [22]

$$
\mathcal{Z}^{(n)}(\vec{P} \mid z)=\sum_{k=0}^{\infty} z^{k} \sum_{\substack{\vec{Y} \\|\vec{Y}|=k}} \prod_{i, j=1}^{n} \prod_{s \in Y_{i}} \frac{1}{E_{Y_{i}, Y_{j}}\left(s \mid P_{i}-P_{j}\right)\left(Q-E_{Y_{i}, Y_{j}}\left(s \mid P_{i}-P_{j}\right)\right)},
$$

where the sum is over all sets of $n$ Young diagrams $\vec{Y}=\left(Y_{1}, \ldots, Y_{n}\right), k=|\vec{Y}|$ is the total number of boxes in $\vec{Y}, \vec{P}=\left(P_{1}, \ldots, P_{n}\right)$ is the vev of the adjoint scalar, $s$ denotes a box in the Young diagram $Y_{i}$, and

$$
E_{Y, W}(P \mid s)=P-l_{W}(s) b^{-1}+\left(a_{Y}(s)+1\right) b,
$$

where $a_{Y}(s)$ and $l_{Y}(s)$ is the arm and the leg length respectively, i.e. the number of boxes in $Y$ to the right and below the box $s \in Y$, see the figure 1 .
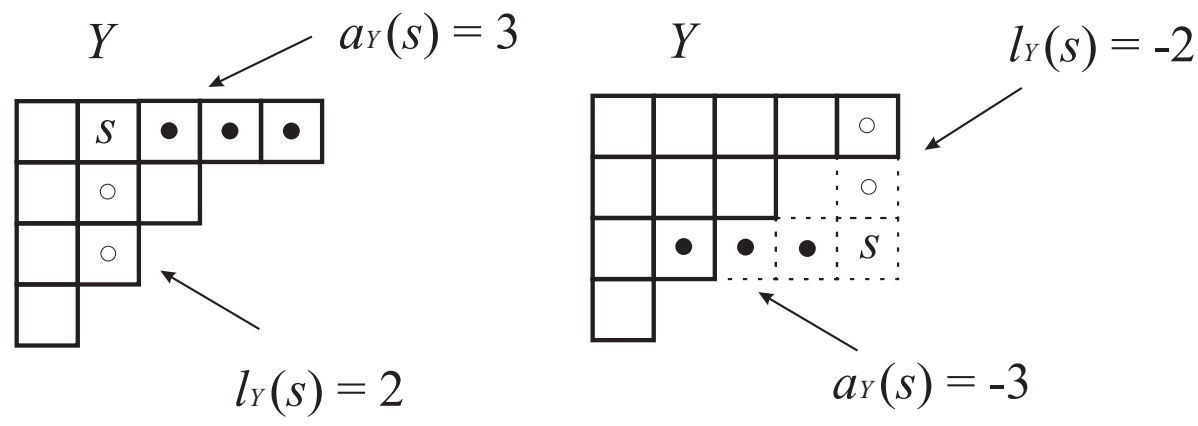

Figure 1: The leg $l_{Y}(s)$ and the arm $a_{Y}(s)$ of the Young diagrams.

In order to compare the answers from both gauge and conformal field theories and for further convenience we have already passed from the deformation parameters $\epsilon_{1}, \epsilon_{2}[23,24$ to the parameter $b$, which parameterize the central charge $c$ in conformal field theory:

$$
\epsilon_{1}=b^{-1}, \quad \epsilon_{2}=b
$$

and $Q=\epsilon_{1}+\epsilon_{2}=b+b^{-1}$.

In the case of instantons on $\mathbb{R}^{4} / \mathbb{Z}_{p}$, we have the similar structure of the partition function, but with some differences, which we are going to describe now. One ascribes a $\mathbb{Z}_{p}$ charge $q_{i}, i=1, \ldots, n$ to each Young diagram $Y_{i}$, where $q_{i}$ can take values $0,1, \ldots, p-1$. We denote this as $Y_{i}^{q_{i}}$. It is convenient to color the Young diagrams in $p$ colors as follows: the box with coordinates $(i, j)$ of the Young diagram $Y^{q}$ has $r \equiv q+i-j \bmod p$ color, see for example the figure 2 . 


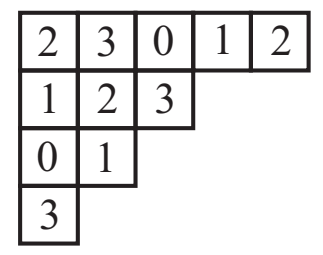

Figure 2: The colored Young diagram $Y=\{5,3,2,1\}$ with the charge $q=2$ and for the case of $p=4$.

Then we introduce two $p$-dimensional vectors $\left\{n_{r}\right\}$ and $\left\{k_{r}\right\}$, where $r=0,1, \ldots, p-1$. The integer $n_{r}$ is the number of Young diagrams with the charge $q=r$ and $k_{r}$ is the number of boxes with color $r$ in all Young diagrams $\left(Y_{1}^{q_{1}}, \ldots, Y_{n}^{q_{n}}\right)$. Thus $\sum_{r} n_{r}=n$ and $\sum_{r} k_{r}=k$.

Vectors $\left\{n_{r}\right\}$ and $\left\{k_{r}\right\}$ are related with topological characteristics of instantons:

$$
c_{1}(E)=\sum_{r=0}^{p-1}\left(n_{r}-2 k_{r}+k_{r+1}+k_{r-1}\right) c_{1}\left(T_{r}\right),
$$

where $c_{1}(E)$ is the first Chern class of gauge bundle $E$ and $c_{1}\left(T_{r}\right)$ is the first Chern class of vector bundle $T_{r}$ on ALE space. The first Chern class $c_{1}\left(T_{r}\right)$ of the $T_{r}$ bundles, $r \neq 0\left(c_{1}\left(T_{0}\right)=0\right)$, forms a basis of the second cohomology group. In this paper we only consider the case $c_{1}(E)=0$. Therefore we obtain the equations

$$
0=n_{r}-2 k_{r}+k_{r+1}+k_{r-1}, \quad \text { for } \quad r=1, \ldots, p-1 .
$$

For simplicity we make the shift $11 k_{r}=k_{0}+\delta k_{r}$, then we have for $\delta k_{r}$

$$
\delta k_{r}=\sum_{l=1}^{p-1} C_{r l} n_{l}, \quad \text { where } \quad C_{r l}=\min (r, l)-\frac{r l}{p} .
$$

Below we will consider only the case of $n=2$. It is clear that $n_{r}$ and $k_{r}$ are integers. Due to this constraints there are following variants of vector $\left\{n_{r}\right\}$ : the first variant: $n_{0}=2, n_{r}=0, r>0$; the second: $n_{r}=n_{p-r}=1$ for $r=1, \ldots,\left\lfloor\frac{p}{2}\right\rfloor$; and the third $n_{p / 2}=2$ in case of $p$ even. Therefore for the first few values of $p$ we have the following series:

\begin{tabular}{|c|c|c||c|c|}
\hline$p=2$ & $\left\{n_{r}\right\}$ & $\left\{\delta k_{r}\right\}$ & $q_{1}$ & $q_{2}$ \\
\hline 1. & $(2,0)$ & $(0,0)$ & 0 & 0 \\
\hline 2. & $(0,2)$ & $(1,1)$ & 1 & 1 \\
\hline \hline$p=3$ & $\left\{n_{r}\right\}$ & $\left\{\delta k_{r}\right\}$ & $q_{1}$ & $q_{2}$ \\
\hline 1. & $(2,0,0)$ & $(0,0,0)$ & 0 & 0 \\
\hline 2. & $(0,1,1)$ & $(0,1,1)$ & 1 & 2 \\
\hline \hline$p=4$ & $\left\{n_{r}\right\}$ & $\left\{\delta k_{r}\right\}$ & $q_{1}$ & $q_{2}$ \\
\hline 1. & $(2,0,0,0)$ & $(0,0,0,0)$ & 0 & 0 \\
\hline 2. & $(0,1,0,1)$ & $(0,1,1,1)$ & 1 & 3 \\
\hline 3. & $(0,0,2,0)$ & $(0,1,2,1)$ & 2 & 2 \\
\hline
\end{tabular}

\footnotetext{
${ }^{1}$ One should notice that $\delta k_{r}$ is the difference between the number of boxes with the color $r$ and with the color 0 in all Young diagrams.
} 
We use the vector symbol $\vec{P}$ to stand for pairs $\vec{P}=(P,-P)$ and $\vec{P}^{\prime}=\left(P^{\prime},-P^{\prime}\right)$ and also $\overrightarrow{Y^{q}}=\left(Y_{1}^{q_{1}}, Y_{2}^{q_{2}}\right)$ and $\vec{W}^{u}=\left(W_{1}^{u_{1}}, W_{2}^{u_{2}}\right)$. Now we can define the contribution of the bifundamental multiplet for $S U(2)$ gauge theory on $\mathbb{R}_{4} / \mathbb{Z}_{p}(p=1$ case see for instance in [1]):

$$
Z_{\mathrm{bif}}^{(p)}\left(\alpha \mid \vec{P}^{\prime}, \overrightarrow{W^{u}}, \vec{P}, \overrightarrow{Y^{q}}\right)=\prod_{i, j=1}^{2} \prod_{s \in Y_{i}^{q_{i}}}\left(Q-E_{Y_{i}, W_{j}}\left(P_{i}-P_{j}^{\prime} \mid s\right)-\alpha\right) \prod_{t \in W_{j}^{u_{j}}}\left(E_{W_{j}, Y_{i}}\left(P_{j}^{\prime}-P_{i} \mid t\right)-\alpha\right),
$$

where

$$
E_{Y, W}(P \mid s)=P-l_{W}(s) b^{-1}+\left(a_{Y}(s)+1\right) b
$$

and the product is over $s \in Y_{i}^{q_{i}}$ and $t \in W_{j}^{u_{j}}$ is restricted to the set $\diamond$ that includes all $s, t$ which satisfy

$$
\begin{aligned}
& s \in Y_{i}^{q_{i} \diamond}: l_{W_{j}}(s)+a_{Y_{i}}(s)+1 \equiv u_{j}-q_{i} \bmod p, \\
& t \in W_{j}^{u_{j} \diamond}: l_{Y_{i}}(t)+a_{W_{j}}(t)+1 \equiv q_{i}-u_{j} \bmod p,
\end{aligned}
$$

(here we also have passed from gauge theory notations to the conformal theory ones, and denote the hypermultiplet mass by $\alpha$.) The partition function for the theory with two fundamental and two antifundamental matter hypermultiplets, which one should compare with the four-point conformal blocks, reads

$$
\mathcal{Z}^{(p)}\left(P_{1}, \alpha_{1}, \alpha_{2}, P_{2}|P| z\right)=\sum_{\left\{\overrightarrow{Y^{q}}\right\}} \frac{Z_{\mathrm{bif}}^{(p)}\left(\alpha_{1} \mid \vec{P}_{1},\left(\varnothing^{0}, \varnothing^{0}\right), \vec{P}, \overrightarrow{Y^{q}}\right) Z_{\mathrm{bif}}^{(p)}\left(\alpha_{2} \mid \vec{P}, \overrightarrow{Y^{q}}, \vec{P}_{2},\left(\varnothing^{0}, \varnothing^{0}\right)\right)}{Z_{\mathrm{bif}}^{(p)}\left(0 \mid \vec{P}, \overrightarrow{Y^{q}}, \vec{P}, \overrightarrow{Y^{q}}\right)} \cdot z^{\frac{\left|\vec{Y}^{q}\right|}{p}}
$$

where the sum goes over all the pairs of Young diagrams $\left\{\overrightarrow{Y^{q}}\right\}$ from the special series, which satisfy to the relation (2.5).

In the next section we perform explicit calculations of the partition function (2.10) in case of $p=4$. As we can see from the table (2.7) there are three series in this case. The first $\left(k=\sum_{r} k_{r}=\left|\overrightarrow{Y^{q}}\right|=4 k_{0}\right)$ and the third $\left(k=4 k_{0}+4\right)$ series make the contribution to the integer powers of parameter $z$, whereas the second series $\left(k=4 k_{0}+3\right)$ gives $z^{k_{0}+3 / 4}$. Thus one can rewrite (2.10) for $p=4$ as

$$
\mathcal{Z}^{(4)}\left(P_{1}, \alpha_{1}, \alpha_{2}, P_{2}|P| z\right)=\sum_{i=1}^{3} \mathcal{Z}^{(4, i \text { series })}\left(P_{1}, \alpha_{1}, \alpha_{2}, P_{2}|P| z\right)
$$

where

$$
\mathcal{Z}^{(4, i \text { series })}\left(P_{1}, \alpha_{1}, \alpha_{2}, P_{2}|P| z\right)=\sum_{\left\{\overrightarrow{Y^{q}}\right\} \in i \text { series }} \frac{Z_{\mathrm{bif}}^{(4)}\left(\alpha_{1} \mid \vec{P}_{1},\left(\varnothing^{0}, \varnothing^{0}\right), \vec{P}, \overrightarrow{Y^{q}}\right) Z_{\mathrm{bif}}^{(4)}\left(\alpha_{2} \mid \vec{P}, \overrightarrow{Y^{q}}, \vec{P}_{2},\left(\varnothing^{0}, \varnothing^{0}\right)\right)}{Z_{\mathrm{bif}}^{(4)}\left(0 \mid \vec{P}, \overrightarrow{Y^{q}}, \vec{P}, \overrightarrow{Y^{q}}\right)} \cdot z^{\frac{\left|\vec{Y}^{q}\right|}{p}}
$$

where $i=1,2,3$. We can also rewrite it as

$$
\mathcal{Z}^{(4)}\left(P_{1}, \alpha_{1}, \alpha_{2}, P_{2}|P| z\right)=\sum_{k_{0}=0}^{\infty} \mathcal{Z}_{k_{0}}^{(4,1 \text { st series })} z^{k_{0}}+\sum_{k_{0}=0}^{\infty} \mathcal{Z}_{k_{0}+3 / 4}^{(4,2 \text { nd series })} z^{k_{0}+3 / 4}+\sum_{k_{0}=0}^{\infty} \mathcal{Z}_{k_{0}+1}^{(4,3 \text { rd series })} z^{k_{0}+1} .
$$

We check that each of these series coincide with the particular type of conformal blocks of $S_{3}$-parafermion algebra up to the $U(1)$-factor which is equal to $(1-z)^{A_{4}}$, where $A_{4}=\frac{1}{2} \alpha_{1}\left(Q-\alpha_{2}\right)$ in case of $p=4$. As we will argue in Section 6 in the case of arbitrary $p$ it should has the same structure with $A_{p}=\frac{2}{p} \alpha_{1}\left(Q-\alpha_{2}\right)$. 


\section{Instanton contributions corresponding to the $S$ and $D$ modules of the $S_{3}$ parafermion algebra}

In this section we make the explicit calculations of the partition function $\mathcal{Z}^{(p)}\left(P_{1}, \alpha_{1}, \alpha_{2}, P_{2}|P| z\right)$ for the case of $p=4$ up to the level $z^{2}$, therefore we need to consider all Young diagrams which contribute to the partition function at the levels $z^{3 / 4}, z, z^{7 / 4}$ and $z^{2}$. For simplicity of the answers we will use the notations from the conformal field theory side, such as

$$
\Delta_{P_{i}}=\frac{1}{4}\left(\frac{Q^{2}}{4}-P_{i}^{2}\right), \quad \Delta_{\alpha_{i}}=\frac{1}{4} \alpha_{i}\left(Q-\alpha_{i}\right), \quad c=2+\frac{3}{2} Q^{2}, \quad Q=b+b^{-1}
$$

- The pairs of the Young diagrams which contribute to the partition function at the level $z^{3 / 4}$ are $(\{3\},\{\varnothing\}),(\{2\},\{1\}),(\{1\},\{1,1\}),(\{\varnothing\},\{1,1,1\})$, which are from the second series with the charges $q_{1}=1, q_{2}=3$. They are explicitly shown in the figure 3 .

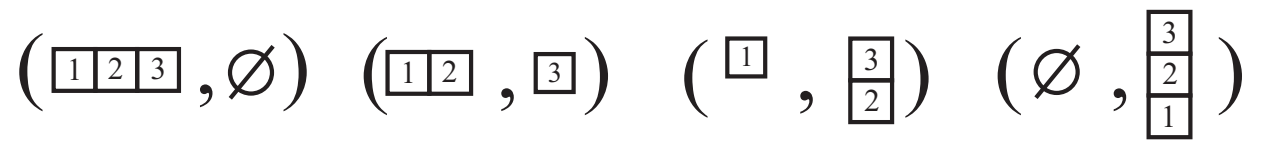

Figure 3: The Young diagrams from the 2nd series at the level $z^{3 / 4}$.

Using the formulas (2.12) and (2.8) for $p=4$ we have

$$
\mathcal{Z}_{3 / 4}^{(4,2 \text { nd series })}=\frac{4}{\left(Q+2 b^{-1}-2 P\right)(Q+2 b+2 P)} .
$$

Notice that there is no symmetry between the pairs of the Young diagrams $\left(Y_{1}^{q_{1}}, Y_{2}^{q_{2}}\right) \leftrightarrow\left(Y_{2}^{q_{1}}, Y_{1}^{q_{2}}\right)$ due to the presence of different charges: $q_{1} \neq q_{2}$, thus $\mathcal{Z}^{(4,2 \text { nd series })}\left(P_{1}, \alpha_{1}, \alpha_{2}, P_{2}|P| z\right)$ doesn't obey $P \rightarrow-P$ symmetry. In other words, the following general identity holds

$$
\mathcal{Z}_{q_{1}, q_{2}}^{(p, i \text { series })}\left(P_{1}, \alpha_{1}, \alpha_{2}, P_{2}|P| z\right)=\mathcal{Z}_{q_{2}, q_{1}}^{(p, i \text { series })}\left(P_{1}, \alpha_{1}, \alpha_{2}, P_{2}|-P| z\right)
$$

where $q_{1}$ and $q_{2}$ are the charges of the Young diagrams of $i$ series. This structure has the clear meaning from the conformal field theory (CFT) point of view.

- The pairs of the Young diagrams which contribute to the partition function at the level $z$ are divided into the first and the third series. For the first series we have the following pairs of the Young diagrams: $(\{4\},\{\varnothing\}),(\{3,1\},\{\varnothing\}),(\{2,1,1\},\{\varnothing\}),(\{1,1,1,1\},\{\varnothing\}),(\{\varnothing\},\{4\}),(\{\varnothing\},\{3,1\}),(\{\varnothing\},\{2,1,1\})$, ( $\{\varnothing\},\{1,1,1,1\})$ with the charges $q_{1}=q_{2}=0$. They are explicitly shown in the figure 4 ,

And we have for $\mathcal{Z}_{1}^{(4,1 \text { st series })}$ the following answer

$$
\mathcal{Z}_{1}^{(4,1 \text { st series })}=\frac{\left(\Delta_{P}-\Delta_{P_{1}}+\Delta_{\alpha_{1}}\right)\left(\Delta_{P}-\Delta_{P_{2}}+\Delta_{\alpha_{2}}\right)}{2 \Delta_{P}}-\frac{1}{2} \alpha_{1}\left(Q-\alpha_{2}\right) .
$$

(we also imply that $\mathcal{Z}_{0}^{(4,1 \text { st series) }}=1$, and there is only one pair of the Young diagrams $\left(\left\{\varnothing^{0}\right\},\left\{\varnothing^{0}\right\}\right)$ for this case). The pairs of the Young diagrams for the third series are $(\{2,2\},\{\varnothing\}),(\{2\},\{1,1\})$, $(\{2,1\},\{1\}),(\{\varnothing\},\{2,2\}),(\{1\},\{2,1\}),(\{1,1\},\{2\})$, with the charges $q_{1}=q_{2}=2$. These diagrams are shown in the figure 5 . 


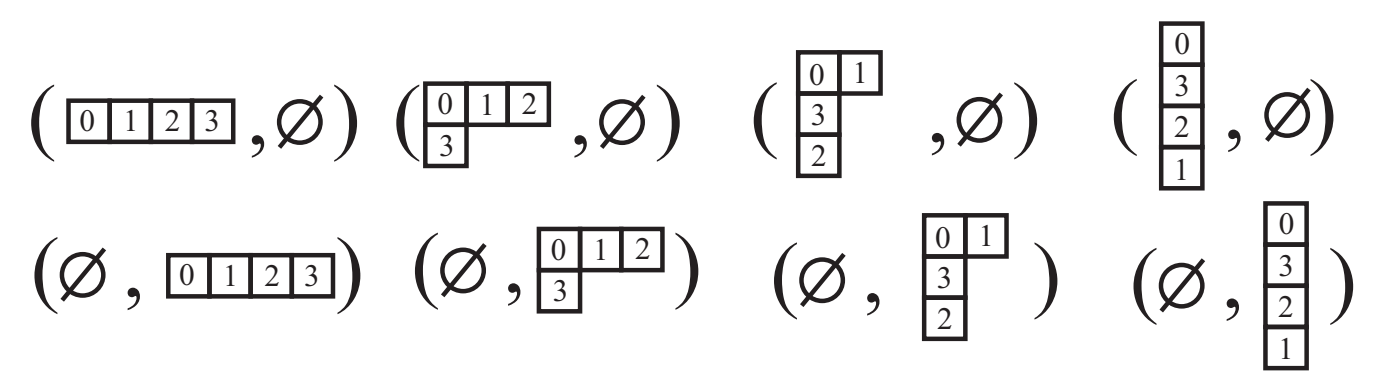

Figure 4: The Young diagrams from the 1st series for the level $z$.

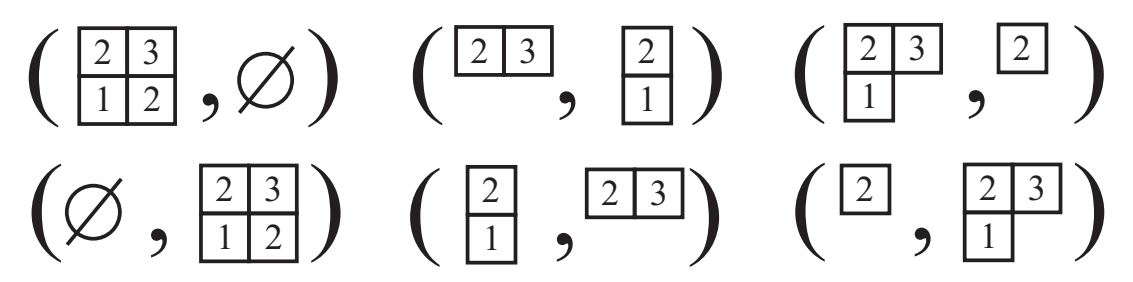

Figure 5: The Young diagrams from the 3rd series for the level $z$.

Then one can obtain

$$
\mathcal{Z}_{1}^{(4,3 \text { rd series })}=\frac{3}{128 \Delta_{P}\left(\Delta_{P}+\frac{c}{8}-\frac{1}{4}\right)} .
$$

- There are 32 pairs of the Young diagrams which contribute to the partition function at the level $z^{7 / 4}$. The first few such diagrams are shown in the figure 6.

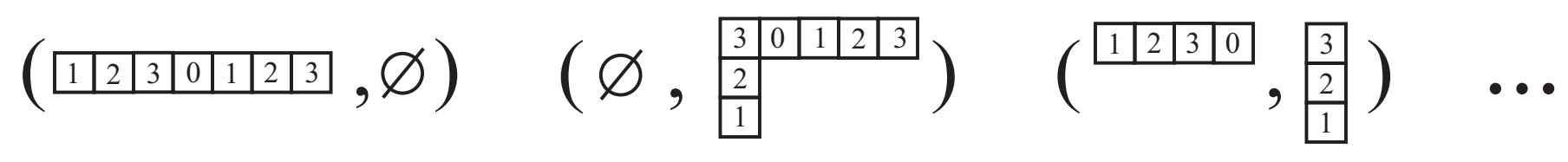

Figure 6: The Young diagrams from the 2nd series for the level $z^{7 / 4}$.

The answer for $\mathcal{Z}_{7 / 4}^{(4,2 \text { nd series })}$ is too cumbersome and we don't show it in this paper explicitly.

- For the level $z^{2}$ the 1st series and the 3rd series give contribution. There are 56 pairs of the Young diagrams from the 1st series and 48 pairs of the Young diagrams from the 3rd series on this level. The explicit answers for $\mathcal{Z}_{2}^{(4,1 \text { st series })}$ and for $\mathcal{Z}_{2}^{(4,3 r d \text { series })}$ are also omitted due to their enormous sizes.

\section{Conformal blocks of the $S_{3}$ parafermion algebra}

\subsection{The extended symmetry algebra of $p=4$ parafermions}

It is known that the extended symmetry algebra of the so called $S_{3}$ parafermions is the spin $4 / 3$ algebra. One can find a detailed discussion of this algebra in [19,25]. Here we point out only the aspects relevant for our discussion. First we write down the Operator Product Expansions (OPEs) of the stress tensor of this algebra $T(z)$ and the fractional spin $4 / 3$ currents $G^{ \pm}(z)$ 


$$
\begin{aligned}
& T(z) T(w)=\frac{c}{2(z-w)^{4}}+\frac{2}{(z-w)^{2}} T(w)+\frac{1}{z-w} \partial T(w)+\cdots, \\
& T(z) G^{ \pm}(w)=\frac{4}{3(z-w)^{2}} G^{ \pm}(w)+\frac{1}{z-w} \partial G^{ \pm}(w)+\cdots, \\
& G^{ \pm}(z) G^{ \pm}(w)=\frac{\lambda^{ \pm}}{(z-w)^{\frac{4}{3}}} G^{\mp}(w)+\frac{\lambda^{\mp}}{(z-w)^{\frac{1}{3}}} \partial G^{\mp}(w)+\cdots, \\
& G^{ \pm}(z) G^{\mp}(w)=\frac{3 c}{8(z-w)^{\frac{8}{3}}}+\frac{1}{(z-w)^{\frac{2}{3}}} T(w)+\cdots,
\end{aligned}
$$

where

$$
c=2+\frac{3}{2} Q^{2}, \quad \lambda^{ \pm}= \pm \sqrt{\frac{c-8}{6}}, \quad Q=b+b^{-1}
$$

and dots stand for non-singular terms. The so called $\mathbb{Z}_{3}$-charge is associated to each field in the above algebra. One ascribes the $\mathbb{Z}_{3}$-charge $q=0$ to the stress tensor $T(z)$ and the $\mathbb{Z}_{3}$-charges $q= \pm 1$ to the fractional currents $G^{ \pm}(z)$.

The next important thing to point out is the moding of the fractional currents. These modes can be determined only acting on the state $\chi_{q}(0)$ with the appropriate $\mathbb{Z}_{3}$-charge $q$

$$
G_{k-\frac{1 \mp q}{3}}^{ \pm} \chi_{q}(0) \stackrel{\text { def }}{=} \oint_{\gamma} \frac{d z}{2 \pi i} z^{k \pm \frac{q}{3}} G^{ \pm}(z) \chi_{q}(0)
$$

where $\gamma$ is a contour encircling the origin and $k \in \mathbb{Z}$. From the formula (4.6) one can extract the rule that says which modes of $G^{ \pm}$can act on the state with the $\mathbb{Z}_{3}$-charge $q$ :

$$
G_{k-\frac{1 \mp q}{3}}^{ \pm}|q\rangle, \quad k \in \mathbb{Z}, \quad q=0, \pm 1
$$

The commutation relations for $T(z)$ and $G^{ \pm}(z)$ modes can be easily obtained from the corresponding OPEs (4.1) and (4.2)

$$
\begin{aligned}
& {\left[L_{m}, L_{n}\right]=(m-n) L_{m+n}+\frac{c}{12}\left(m^{3}-m\right) \delta_{m+n, 0},} \\
& {\left[L_{m}, G_{r}^{ \pm}\right]=\left(\frac{m}{3}-r\right) G_{m+r}^{ \pm},}
\end{aligned}
$$

where $m, n \in \mathbb{Z}$ and $r=k+\frac{1 \mp q}{3}$ with $k \in \mathbb{Z}$ and $q$ the $\mathbb{Z}_{3}$-charge of the state on which the corresponding $G_{r}^{ \pm}$acts. It appears that due to the fact that the OPEs (4.3) and (4.4) of the fractional spin algebra contain the fractional powers, it is not possible to obtain the ordinary commutation relations but it is possible to write down the so called generalized commutation relations

$$
\begin{aligned}
& \sum_{k=0}^{+\infty} C_{k}^{\left(-\frac{2}{3}\right)}\left(G_{\frac{q}{3}+n-k}^{+} G_{\frac{2+q}{3}+m-k}^{+}-G_{\frac{q}{3}+m-k}^{+} G_{\frac{2+q}{3}+n-k}^{+}\right)|q\rangle=\frac{\lambda^{+}}{2}(n-m) G_{\frac{2+2 q}{3}+n+m}^{+}|q\rangle \\
& \sum_{k=0}^{+\infty} C_{k}^{\left(-\frac{2}{3}\right)}\left(G_{-\frac{q}{3}+n-k}^{-} G_{\frac{2-q}{3}+m-k}^{-}-G_{-\frac{q}{3}+m-k}^{-} G_{\frac{2-q}{3}+n-k}^{+}\right)|q\rangle=\frac{\lambda^{-}}{2}(n-m) G_{\frac{2-2 q}{3}+n+m}^{+}|q\rangle \\
& \sum_{k=0}^{+\infty} C_{k}^{\left(-\frac{1}{3}\right)}\left(G_{\frac{1+q}{3}+n-k}^{+} G_{-\frac{1+q}{3}+m-k}^{-}+G_{\frac{1+q}{3}+m-k}^{-} G_{-\frac{1+q}{3}+n-k}^{+}\right)|q\rangle=\left(L_{n+m}+\frac{3 c}{16}\left(n+1+\frac{q}{3}\right)\left(n+\frac{q}{3}\right)\right)|q\rangle
\end{aligned}
$$


where $m, n \in \mathbb{Z}$ and

$$
C_{k}^{(\nu)}=(-1)^{k}\left(\begin{array}{c}
\nu \\
k
\end{array}\right)=\frac{(-1)^{k}}{k !} \prod_{i=1}^{k}(\nu-i+1) .
$$

The structure of the highest weight states in this algebra is the following. There are three different modules denoted by $S, D$ and $R$. The highest weight states in these modules are annihilated by the generators $L_{m}$ and $G_{r}^{ \pm}$with $r, m>0$. The $R$-module is not relevant to our discussion. We denote the primary states as

$$
|\alpha ; q\rangle
$$

where $\alpha$ is the Liouville parameter of the state and $q$ is the $\mathbb{Z}_{3}$-charge of the state. The conjugated state is denoted by $\langle\alpha ; q|$. Then in these notations the highest weight state in the $S$-module is $|\alpha ; 0\rangle$. In the $D$-module there are two highest weight states, i.e the highest weight state is double degenerate, and they are denoted by $|\alpha ; \pm 1\rangle$. The conformal dimensions of these highest weight states are

$$
\Delta_{\alpha}^{(s)}=\frac{1}{4} \alpha(Q-\alpha), \quad \Delta_{\alpha}^{(d)}=\frac{1}{4} \alpha(Q-\alpha)+\frac{1}{12} .
$$

We use the following convention for the highest weight states in the $D$-module

$$
G_{0}^{ \pm}|\alpha ; \pm 1\rangle=\Lambda^{ \pm}|\alpha ; \mp 1\rangle, \quad \Lambda^{ \pm}= \pm \sqrt{\frac{c}{24}-\Delta_{\alpha}^{(d)}} .
$$

The basis of states in each module can be chosen in the following form

$$
\prod_{i=1}^{u} G_{-r_{i}}^{ \pm} \prod_{j=1}^{v} L_{-n_{j}}|\alpha ; q\rangle .
$$

The level of the state is defined as $l=l_{0}+\delta$ with $l_{0}=\sum_{i=1}^{u} r_{i}+\sum_{j=1}^{v} n_{j}$ and where $\delta=0$ for the $S$-module and $\delta=\frac{1}{12}$ for the $D$-module. Note that in these definitions the level of the highest weight state in the $D$-module is $\frac{1}{12}$ and its dimension is $\Delta_{\alpha}^{(d)}=\Delta_{\alpha}^{(s)}+\frac{1}{12}$, which means that we count the level of the state off the level of the highest weight state in the $S$-module. Taking into account the rule (4.7) for acting on the states one can show that the possible values of the levels in the $S$-module are $l \in \mathbb{Z}_{\geqslant 0}$ and $l \in \mathbb{Z}_{\geqslant 0}+\frac{1}{3}$. The same in the $D$-module are $l \in \mathbb{Z}_{\geqslant 0}+\frac{1}{12}$ and $l \in \mathbb{Z}_{\geqslant 0}+\frac{3}{4}$. The hermitian conjugated generators are: $\left(L_{n}\right)^{\dagger}=L_{-n},\left(G_{r}^{ \pm}\right)^{\dagger}=-G_{-r}^{\mp}$.

\subsection{Conformal blocks}

To calculate the coefficients of the parafermionic conformal blocks we evaluate the four-point correlation function of the highest weight states in the $S$-module. We denote the vertex operator of this highest weight state as $W_{\alpha}^{(s)}(z)$. Then the object of our interest is

$$
\left\langle m_{1} ; 0\left|W_{\alpha_{1}}^{(s)}(1) W_{\alpha_{2}}^{(s)}(z)\right| m_{2} ; 0\right\rangle \quad\left(\equiv\left\langle W_{m_{1}}^{(s)}(\infty) W_{\alpha_{1}}^{(s)}(1) W_{\alpha_{2}}^{(s)}(z) W_{m_{2}}^{(s)}(0)\right\rangle\right) .
$$

Here we already use the notations of the Liouville parameters like in the gauge theory to match the conformal block to the partition function. The connection of the parameters $\alpha, m_{1}$ and $m_{2}$ with the gauge theory parameter is

$$
\alpha=\frac{Q}{2}+P, \quad m_{1}=\frac{Q}{2}+P_{1}, \quad m_{2}=\frac{Q}{2}+P_{2} .
$$


We evaluate this correlation function by inserting the complete set of states at each level

$$
\hat{\mathbf{1}}_{l}=\sum_{i, j}|i\rangle_{l} \times\left(K_{\alpha}^{-1}(l)\right)_{i j} \times{ }_{l}\langle j|
$$

where $\left\{|1\rangle_{l},|2\rangle_{l}, \ldots\right\}$ is the set of the descendants of primary fields at the level $l$ and $K_{\alpha}^{-1}(l)$ is the inverse Gram/Shapovalov matrix at the level $l\left(\left(K_{\alpha}(l)\right)_{i j}={ }_{l}\langle i \mid j\rangle_{l}\right)$. We notice that descedants states at each level depend on Liouville parameter $\alpha$ as well as the Gram/Shapovalov matrix. The corresponding conformal block is represented as the series in the fractional powers $l \in \mathbb{Z}_{\geqslant 0}, l \in \mathbb{Z}_{\geqslant 0}+\frac{1}{12}, l \in \mathbb{Z}_{\geqslant 0}+\frac{1}{3}$ and $l \in \mathbb{Z}_{\geqslant 0}+\frac{3}{4}$ of $z$. But as we already know from the gauge theory partition function (2.13) that it doesn't contain the powers of the expansion parameter $l \in \mathbb{Z}_{\geqslant 0}+\frac{1}{12}$ and $l \in \mathbb{Z}_{\geqslant 0}+\frac{1}{3}$. Then we make a conclusion that to match the gauge theory result we must only consider the contributions to the correlation function (4.16) arising from the introduction of the complete sets (4.18) of states at the levels $l \in \mathbb{Z}_{\geqslant 0}$ and $l \in \mathbb{Z}_{\geqslant 0}+\frac{3}{4}$. As we will see further there are two types of conformal blocks on the levels $l \in \mathbb{Z}_{\geqslant 0}$ :

$$
\begin{aligned}
\sum_{l=0,1,2, \ldots} & \sum_{i, j}\left\langle m_{1} ; 0\left|W_{\alpha_{1}}^{(s)}(1)\right| i\right\rangle_{l} \times\left(K_{\alpha}^{-1}(l)\right)_{i j} \times{ }_{l}\left\langle j\left|W_{\alpha_{2}}^{(s)}(z)\right| m_{2} ; 0\right\rangle= \\
= & C_{m_{1}, \alpha_{1}}^{\alpha} C_{\alpha, \alpha_{2}}^{m_{2}} \times z^{\Delta_{\alpha}^{(s)}-\Delta_{\alpha_{2}}^{(s)}-\Delta_{m_{2}}^{(s)}} \mathcal{F}^{(1)}\left(\Delta_{m_{1}}^{(s)}, \Delta_{\alpha_{1}}^{(s)}, \Delta_{\alpha_{2}}^{(s)}, \Delta_{m_{2}}^{(s)}\left|\Delta_{\alpha}^{(s)}\right| z\right)+ \\
& \quad+\tilde{C}_{m_{1}, \alpha_{1}}^{\alpha} \tilde{C}_{\alpha, \alpha_{2}}^{m_{2}} \times z^{\Delta_{\alpha}^{(s)}-\Delta_{\alpha_{2}}^{(s)}-\Delta_{m_{2}}^{(s)}} \mathcal{F}^{(3)}\left(\Delta_{m_{1}}^{(s)}, \Delta_{\alpha_{1}}^{(s)}, \Delta_{\alpha_{2}}^{(s)}, \Delta_{m_{2}}^{(s)}\left|\Delta_{\alpha}^{(s)}\right| z\right)
\end{aligned}
$$

where

$$
\begin{aligned}
& C_{m_{1}, \alpha_{1}}^{\alpha}=\left\langle m_{1} ; 0\left|W_{\alpha_{1}}^{(s)}(1)\right| \alpha ; 0\right\rangle \\
& \tilde{C}_{m_{1}, \alpha_{1}}^{\alpha}=\left\langle m_{1} ; 0\left|\tilde{W}_{\alpha_{1}}^{(s)}(1)\right| \alpha ; 0\right\rangle
\end{aligned}
$$

are the structure constants and $\tilde{W}_{\alpha}^{(s)}(z)$ is the vertex operator corresponding to the state

$$
\left|\tilde{W}_{\alpha}^{(s)}\right\rangle=\left(G_{-\frac{2}{3}}^{+} G_{-\frac{1}{3}}^{-}-G_{-\frac{2}{3}}^{-} G_{-\frac{1}{3}}^{+}\right)|\alpha ; 0\rangle
$$

is the descendant that is also a Virasoro primary state. We see that there are two types of conformal blocks due to the fact that there are two different types of structure constants: one for the field $W_{\alpha}^{(s)}(z)$ and the other for the field $\tilde{W}_{\alpha}^{(s)}(z)$ and these structure constants cannot be expressed through each other. We denote the conformal blocks corresponding to $W$ and $\tilde{W}$ as $\mathcal{F}^{(1)}$ and $\mathcal{F}^{(3)}$ respectively. Note that the expansion in the second conformal block starts from the first power of $z$.

The same thing can be performed at the levels $l \in \mathbb{Z}_{\geqslant 0}+\frac{3}{4}$

$$
\begin{aligned}
\sum_{l=\frac{3}{4}, \frac{7}{4}, \frac{11}{4}, \ldots} & \sum_{i, j}\left\langle m_{1} ; 0\left|W_{\alpha_{1}}^{(s)}(1)\right| i\right\rangle_{l} \times\left(K_{\alpha}^{-1}(l)\right)_{i j} \times{ }_{l}\left\langle j\left|W_{\alpha_{2}}^{(s)}(z)\right| m_{2} ; 0\right\rangle= \\
= & \mathbb{C}_{m_{1}, \alpha_{1}}^{(+) \alpha} \mathbb{C}_{\alpha, \alpha_{2}}^{(+) m_{2}} \times z^{\Delta_{\alpha}^{(s)}-\Delta_{\alpha_{2}}^{(s)}-\Delta_{m_{2}}^{(s)}} \mathcal{F}^{(2)}\left(\Delta_{m_{1}}^{(s)}, \Delta_{\alpha_{1}}^{(s)}, \Delta_{\alpha_{2}}^{(s)}, \Delta_{m_{2}}^{(s)}|P| z\right)+ \\
& +\mathbb{C}_{m_{1}, \alpha_{1}}^{(-) \alpha} \mathbb{C}_{\alpha, \alpha_{2}}^{(-) m_{2}} \times z^{\Delta_{\alpha}^{(s)}-\Delta_{\alpha_{2}}^{(s)}-\Delta_{m_{2}}^{(s)}} \mathcal{F}^{(2)}\left(\Delta_{m_{1}}^{(s)}, \Delta_{\alpha_{1}}^{(s)}, \Delta_{\alpha_{2}}^{(s)}, \Delta_{m_{2}}^{(s)}|-P| z\right),
\end{aligned}
$$

where $\left\{|i\rangle_{l}\right\}$ is the set of the descendants in the $D$-module at the level $l, K_{\alpha}^{-1}(l)$ is the inverse Gram/Shapovalov matrix at the level $l$ and 


$$
\mathbb{C}_{m_{1}, \alpha_{1}}^{( \pm) \alpha}=\left\langle m_{1} ; 0\left|V_{\alpha_{1}}^{(s)+}(1)\right| \alpha ;-1\right\rangle \pm\left\langle m_{1} ; 0\left|V_{\alpha_{1}}^{(s)-}(1)\right| \alpha ; 1\right\rangle
$$

where $V_{\alpha}^{(s) \pm}(z)$ are the vertex operators corresponding to the states

$$
\left|V_{\alpha}^{(s) \pm}\right\rangle=G_{-\frac{1}{3}}^{ \pm}|\alpha ; 0\rangle
$$

which are the first fractional descendants of the state $|\alpha ; 0\rangle$. Note that the expansion of the conformal block $\mathcal{F}^{(2)}$ starts from $z^{\frac{3}{4}}$. Another important thing one should notice about the conformal block $\mathcal{F}^{(2)}$ is that it is not symmetric under the flip of the sign of $P$ in the parameter $\alpha=\frac{Q}{2}+P$. That's why we write $P$ instead of $\Delta_{\alpha}^{(s)}$ in the formula (4.23).

With the notations (4.20), (4.21) and (4.24) we can briefly summarize the statements (4.19) and (4.23)

$$
\begin{aligned}
& \sum_{l=0,1,2,}^{+\infty} \sum_{i, j}\left\langle m_{1} ; 0\left|W_{\alpha_{1}}^{(s)}(1)\right| i\right\rangle_{l} \times\left(K_{\alpha}^{-1}(l)\right)_{i j} \times{ }_{l}\left\langle i\left|W_{\alpha_{2}}^{(s)}(z)\right| m_{2} ; 0\right\rangle= \\
& =z^{\Delta_{\alpha}^{(s)}-\Delta_{\alpha_{2}}^{(s)}-\Delta_{m_{2}}^{(s)}}\left(C_{m_{1}, \alpha_{1}}^{\alpha} \cdot C_{\alpha, \alpha_{2}}^{m_{2}} \times \mathcal{F}^{(1)}\left(\Delta_{m_{1}}^{(s)}, \Delta_{\alpha_{1}}^{(s)}, \Delta_{\alpha_{2}}^{(s)}, \Delta_{m_{2}}^{(s)}\left|\Delta_{\alpha}^{(s)}\right| z\right)+\right. \\
& \left.+\tilde{C}_{m_{1}, \alpha_{1}}^{\alpha} \cdot \tilde{C}_{\alpha, \alpha_{2}}^{m_{2}} \times \mathcal{F}^{(3)}\left(\Delta_{m_{1}}^{(s)}, \Delta_{\alpha_{1}}^{(s)}, \Delta_{\alpha_{2}}^{(s)}, \Delta_{m_{2}}^{(s)}\left|\Delta_{\alpha}^{(s)}\right| z\right)\right), \\
& \sum_{l=\frac{3}{4}, \frac{7}{4} \frac{11}{4},}^{+\infty} \sum_{i, j}\left\langle m_{1} ; 0\left|W_{\alpha_{1}}^{(s)}(1)\right| i\right\rangle_{l} \times\left(K_{\alpha}^{-1}(l)\right)_{i j} \times{ }_{l}\left\langle j\left|W_{\alpha_{2}}^{(s)}(z)\right| m_{2} ; 0\right\rangle= \\
& =z^{\Delta_{\alpha}^{(s)}-\Delta_{\alpha_{2}}^{(s)}-\Delta_{m_{2}}^{(s)}}\left(\mathbb{C}_{m_{1}, \alpha_{1}}^{(+) \alpha} \cdot \mathbb{C}_{\alpha, \alpha_{2}}^{(+) m_{2}} \times \mathcal{F}^{(2)}\left(\Delta_{m_{1}}^{(s)}, \Delta_{\alpha_{1}}^{(s)}, \Delta_{\alpha_{2}}^{(s)}, \Delta_{m_{2}}^{(s)}|P| z\right)+\right. \\
& \left.+\mathbb{C}_{m_{1}, \alpha_{1}}^{(-) \alpha} \cdot \mathbb{C}_{\alpha, \alpha_{2}}^{(-) m_{2}} \times \mathcal{F}^{(2)}\left(\Delta_{m_{1}}^{(s)}, \Delta_{\alpha_{1}}^{(s)}, \Delta_{\alpha_{2}}^{(s)}, \Delta_{m_{2}}^{(s)}|-P| z\right)\right) .
\end{aligned}
$$

And for the sake of convenience we admit the following notations for the coefficients in the conformal block expansions

$$
\begin{aligned}
& \mathcal{F}^{(1)}\left(\Delta_{m_{1}}^{(s)}, \Delta_{\alpha_{1}}^{(s)}, \Delta_{\alpha_{2}}^{(s)}, \Delta_{m_{2}}^{(s)}\left|\Delta_{\alpha}^{(s)}\right| z\right)=1+\mathcal{F}_{1}^{(1)} z+\mathcal{F}_{2}^{(1)} z^{2}+\cdots, \\
& \mathcal{F}^{(2)}\left(\Delta_{m_{1}}^{(s)}, \Delta_{\alpha_{1}}^{(s)}, \Delta_{\alpha_{2}}^{(s)}, \Delta_{m_{2}}^{(s)}|P| z\right)=\mathcal{F}_{\frac{3}{4}}^{(2)} z^{\frac{3}{4}}+\mathcal{F}_{\frac{7}{4}}^{(2)} z^{\frac{7}{4}}+\cdots \\
& \mathcal{F}^{(3)}\left(\Delta_{m_{1}}^{(s)}, \Delta_{\alpha_{1}}^{(s)}, \Delta_{\alpha_{2}}^{(s)}, \Delta_{m_{2}}^{(s)}\left|\Delta_{\alpha}^{(s)}\right| z\right)=\mathcal{F}_{1}^{(3)} z+\mathcal{F}_{2}^{(3)} z^{2}+\cdots
\end{aligned}
$$

The above discussion drives us to the conclusion that two key ingredients we need to calculate are the inverse Gram/Shapovalov matrix at the particular level and the matrix elements of the states at the particular level

$$
\left\langle m_{1} ; 0\left|W_{\alpha_{1}}^{(s)}(1)\right| i\right\rangle_{l}, \quad{ }_{l}\left\langle j\left|W_{\alpha_{2}}^{(s)}(z)\right| m_{2} ; 0\right\rangle,
$$

where $|i\rangle_{l}$ is a state at the level $l$. To evaluate these matrix elements we must derive the commutation relations for the vertex operators.

The derivation of the commutation relations for $W_{\alpha}^{(s)}(z)$ and $\tilde{W}_{\alpha}^{(s)}(z)$ is presented in the Appendix A. Here we just cite the result 


$$
\begin{aligned}
& {\left[L_{m}, W_{\alpha}^{(s)}(z)\right] }=z^{m} \partial W_{\alpha}^{(s)}(z)+(m+1) \Delta_{\alpha}^{(s)} W_{\alpha}^{(s)}(z) \\
& {\left[G_{r}^{ \pm}, W_{\alpha}^{(s)}(z)\right] }=z^{r+\frac{1}{3}} V_{\alpha}^{(s) \pm}(z) \\
& {\left[L_{m}, V_{\alpha}^{(s) \pm}(z)\right] }=z^{m} \partial V_{\alpha}^{(s) \pm}(z)+(m+1)\left(\Delta_{\alpha}^{(s)}+\frac{1}{3}\right) V_{\alpha}^{(s) \pm}(z) \\
& \sum_{l=0}^{+\infty} C_{l}^{\left(-\frac{1}{3}\right)}\left(z^{l} G_{r-l-\frac{1}{3}}^{ \pm} V_{\alpha}^{(s) \mp}(z)+z^{-l-\frac{1}{3}} V_{\alpha}^{(s) \mp}(z) G_{r+l}^{ \pm}\right)= \\
&=z^{r-\frac{2}{3}}\left(r+\frac{1}{3}\right) \Delta_{\alpha}^{(s)} W_{\alpha}^{(s)}(z)+z^{r+\frac{1}{3}}\left(\frac{1}{2} \partial W_{\alpha}^{(s)}(z) \pm \tilde{W}_{\alpha}^{(s)}(z)\right)
\end{aligned}
$$

Also we derived the commutation relations in the $D$-module in the Appendix $\mathrm{B}$. These relations may be useful for those who want to calculate the matrix elements of the $D$-module fields. Here we again cite only the result

$$
\begin{aligned}
& {\left[L_{m}, W_{\alpha}^{(d) \pm}(z)\right]=z^{m} \partial W_{\alpha}^{(d) \pm}(z)+(m+1) \Delta_{\alpha}^{(d)} W_{\alpha}^{(d) \pm}(z),} \\
& {\left[L_{m}, V_{\alpha}^{(d)( \pm)}(z)\right]=z^{m} \partial V_{\alpha}^{(d)( \pm)}(z)+(m+1)\left(\Delta_{\alpha}^{(d)}+\frac{2}{3}\right) V_{\alpha}^{(d)( \pm)}(z),} \\
& \sum_{l=0}^{+\infty} C_{l}^{\left(-\frac{2}{3}\right)}\left(z^{l} G_{r-l-\frac{2}{3}}^{ \pm} W_{\alpha}^{(d) \pm}(z)-z^{-l-\frac{2}{3}} W_{\alpha}^{(d) \pm}(z) G_{r+l}^{ \pm}\right)= \\
& =z^{r-\frac{2}{3}}\left(r+\frac{1}{3}\right) \Lambda^{ \pm} W_{\alpha}^{(d) \mp}(z)+z^{r+\frac{1}{3}}\left(\frac{2 \Lambda^{ \pm}}{3 \Delta_{\alpha}^{(d)}} \partial W_{\alpha}^{(d) \mp}(z)+\tilde{W}_{\alpha}^{(d) \mp}(z)\right), \\
& \sum_{l=0}^{+\infty} C_{l}^{\left(-\frac{1}{3}\right)}\left(z^{l} G_{r-l-\frac{1}{3}}^{ \pm} W_{\alpha}^{(d) \mp}(z)+z^{-l-\frac{1}{3}} W_{\alpha}^{(d) \mp}(z) G_{r+l}^{ \pm}\right)=\frac{1}{2} z^{r+\frac{1}{3}}\left(V_{\alpha}^{(d)(+)}(z) \pm V_{\alpha}^{(d)(-)}(z)\right), \\
& {\left[G_{r}^{ \pm}, V_{\alpha}^{(d)+}(z)\right]=z^{r-\frac{2}{3}}\left(r+\frac{1}{3}\right)\left(\Delta_{\alpha}^{(d)}+\frac{c}{12}+\lambda^{ \pm} \Lambda^{\mp}\right) W_{\alpha}^{(d) \pm}(z)+} \\
& +z^{r+\frac{1}{3}} \frac{\Delta_{\alpha}^{(d)}+\frac{c}{12}+\lambda^{ \pm} \Lambda^{\mp}}{3 \Delta_{\alpha}^{(d)}} \partial W_{\alpha}^{(d) \pm}(z)-z^{r+\frac{1}{3}}\left(\Lambda^{ \pm}-\frac{1}{2} \lambda^{ \pm}\right) \tilde{W}_{\alpha}^{(d) \pm}(z), \\
& {\left[G_{r}^{ \pm}, V_{\alpha}^{(d)-}(z)\right]=z^{r-\frac{2}{3}}\left(r+\frac{1}{3}\right)\left(\Delta_{\alpha}^{(d)}+\frac{c}{12}-\lambda^{ \pm} \Lambda^{\mp}\right) W_{\alpha}^{(d) \pm}(z) \mp} \\
& \mp z^{r+\frac{1}{3}} \frac{\Delta_{\alpha}^{(d)}+\frac{c}{12}-\lambda^{ \pm} \Lambda^{\mp}}{3 \Delta_{\alpha}^{(d)}} \partial W_{\alpha}^{(d) \pm}(z) \pm z^{r+\frac{1}{3}}\left(\Lambda^{ \pm}+\frac{1}{2} \lambda^{ \pm}\right) \tilde{W}_{\alpha}^{(d) \pm}(z),
\end{aligned}
$$

where $V_{\alpha}^{(d)( \pm)}(z)$ and $\tilde{W}_{\alpha}^{(d) \pm}(z)$ are the vertex operators corresponding respectively to the states

$$
\begin{aligned}
& \left|V_{\alpha}^{(d)( \pm)}\right\rangle=G_{-\frac{2}{3}}^{+}\left|W_{\alpha}^{(d)-}\right\rangle \pm G_{-\frac{2}{3}}^{-}\left|W_{\alpha}^{(d)+}\right\rangle \\
& \left|\tilde{W}_{\alpha}^{(d) \pm}\right\rangle=G_{-1}^{\mp}\left|W_{\alpha}^{(d) \mp}\right\rangle-\frac{2 \Lambda^{\mp}}{3 \Delta_{\alpha}^{(d)}} L_{-1}\left|W_{\alpha}^{(d) \pm}\right\rangle .
\end{aligned}
$$




\subsubsection{Level $3 / 4$}

The basis of states at this level can be chosen in the following way

$$
\begin{aligned}
&|1\rangle_{3 / 4}=G_{-\frac{2}{3}}^{+}|\alpha ;-1\rangle, \\
&|2\rangle_{3 / 4}=G_{-\frac{2}{3}}^{-}|\alpha ; 1\rangle .
\end{aligned}
$$

We must evaluate the following quantity

$$
\sum_{i, j=1}^{2}\left\langle m_{1} ; 0\left|W_{\alpha_{1}}^{(s)}(1)\right| i\right\rangle_{3 / 4} \times\left(K_{\alpha}^{-1}(3 / 4)\right)_{i j} \times_{3 / 4}\left\langle j\left|W_{\alpha_{2}}^{(s)}(z)\right| m_{2} ; 0\right\rangle .
$$

The calculation of the corresponding Gram/Shapovalov matrix gives

$$
K_{\alpha}(3 / 4)=\left(\begin{array}{cc}
-\left(\Delta_{\alpha}^{(d)}+\frac{c}{12}\right) & \sqrt{\frac{c-8}{6}} \sqrt{\frac{c}{24}-\Delta_{\alpha}^{(d)}} \\
\sqrt{\frac{c-8}{6}} \sqrt{\frac{c}{24}-\Delta_{\alpha}^{(d)}} & -\left(\Delta_{\alpha}^{(d)}+\frac{c}{12}\right)
\end{array}\right) .
$$

It is easy to find the inverse Gram/Shapovalov matrix

$$
K_{\alpha}^{-1}(3 / 4)=\left(\begin{array}{cc}
-\frac{2 b\left(1+b\left(2 \alpha+b\left(4+b^{2}+2 b \alpha-2 \alpha^{2}\right)\right)\right)}{(b+\alpha)\left(2+b^{2}-b \alpha\right)\left(1+2 b^{2}-b \alpha\right)(1+b \alpha)} & -\frac{2 b\left|1-b^{2}\right|\left|1+b^{2}-2 b \alpha\right|}{(b+\alpha)\left(2+b^{2}-b \alpha\right)\left(1+2 b^{2}-b \alpha\right)(1+b \alpha)} \\
-\frac{2 b\left|1-b^{2}\right|\left|1+b^{2}-2 b \alpha\right|}{(b+\alpha)\left(2+b^{2}-b \alpha\right)\left(1+2 b^{2}-b \alpha\right)(1+b \alpha)} & -\frac{2 b\left(1+b\left(2 \alpha+b\left(4+b^{2}+2 b \alpha-2 \alpha^{2}\right)\right)\right)}{(b+\alpha)\left(2+b^{2}-b \alpha\right)\left(1+2 b^{2}-b \alpha\right)(1+b \alpha)}
\end{array}\right) .
$$

Using the commutation relations (4.31) we have

$$
\begin{aligned}
\left\langle m_{1} ; 0\left|W_{\alpha_{1}}(1)\right| 1\right\rangle_{3 / 4} & =-\left(\mathbb{C}_{m_{1}, \alpha_{1}}^{(+) \alpha}+\mathbb{C}_{m_{1}, \alpha_{1}}^{(-) \alpha}\right), \\
\left\langle m_{1} ; 0\left|W_{\alpha_{2}}(1)\right| 2\right\rangle_{3 / 4} & =-\left(\mathbb{C}_{m_{1}, \alpha_{1}}^{(+) \alpha}-\mathbb{C}_{m_{1}, \alpha_{1}}^{(-) \alpha}\right), \\
3 / 4\left\langle 1\left|W_{\alpha_{2}}(z)\right| m_{2} ; 0\right\rangle & =-z^{\Delta_{\alpha}^{(s)}-\Delta_{\alpha_{2}}^{(s)}-\Delta_{m_{2}}^{(s)}+\frac{3}{4}}\left(\mathbb{C}_{\alpha, \alpha_{2}}^{(+) m_{2}}+\mathbb{C}_{\alpha, \alpha_{2}}^{(-) m_{2}}\right), \\
3 / 4\left\langle 2\left|W_{\alpha_{2}}(z)\right| m_{2} ; 0\right\rangle & =-z^{\Delta_{\alpha}^{(s)}-\Delta_{\alpha_{2}}^{(s)}-\Delta_{m_{2}}^{(s)}+\frac{3}{4}}\left(\mathbb{C}_{\alpha, \alpha_{2}}^{(+) m_{2}}-\mathbb{C}_{\alpha, \alpha_{2}}^{(-) m_{2}}\right) .
\end{aligned}
$$

Note that there are modules in the non-diagonal matrix elements in the Gram/Shapovalov matrix. To evaluate the contributions to the conformal block we must choose the sign of these modules. It appears that to match the gauge theory result (3.2) we should choose the following sign $\mid 1-b^{2} \| 1+b^{2}-$ $2 b \alpha \mid=\left(b^{2}-1\right)\left(2 b \alpha-1-b^{2}\right)=2 b\left(b^{2}-1\right) P$. Substituting the matrix elements (4.38) and the inverse Gram/Shapovalov matrix (4.37) into (4.35) after some simple algebra one has the contribution to the conformal block $\mathcal{F}^{(2)}\left(\Delta_{m_{1}}^{(s)}, \Delta_{\alpha_{1}}^{(s)}, \Delta_{\alpha_{2}}^{(s)}, \Delta_{m_{2}}^{(s)}|P| z\right)$

$$
\mathcal{F}_{3 / 4}^{(2)}=-\frac{8 b}{(b+\alpha)\left(2+b^{2}-b \alpha\right)}=-\frac{32}{(Q+2 b+P)\left(Q+2 b^{-1}-P\right)} .
$$

One should also notice that the terms proportional to $\mathbb{C}_{m_{1}, \alpha_{1}}^{(+) \alpha} \cdot \mathbb{C}_{\alpha, \alpha_{2}}^{(-) m_{2}}$ or $\mathbb{C}_{m_{1}, \alpha_{1}}^{(-) \alpha} \cdot \mathbb{C}_{\alpha, \alpha_{2}}^{(+) m_{2}}$ does not appear in the final result when we calculate (4.35). This result is in the accordance with the formula (4.23).

The interesting fact is that we can also match the result in case of the different choice of the sign of the non-diagonal elements of the inverse Gram/Shapovalov matrix. If we choose the opposite sign 
$\left|1-b^{2}\right|\left|1+b^{2}-2 b \alpha\right|=-\left(b^{2}-1\right)\left(2 b \alpha-1-b^{2}\right)=-2 b\left(b^{2}-1\right) P$, we obtain the result (4.39) with $P$ replaced by $-P$. Then if we simultaneously exchange the $\mathbb{Z}_{4}$-charges $q_{1}=1$ and $q_{2}=3$ associated to the Young diagrams in the 2nd series in the gauge theory (3.2), our results will again coincide. Or, equivalently, given the particular choice of the sign in the Gram/Shapovalov matrix the conformal block $\mathcal{F}^{(2)}\left(\Delta_{m_{1}}^{(s)}, \Delta_{\alpha_{1}}^{(s)}, \Delta_{\alpha_{2}}^{(s)}, \Delta_{m_{2}}^{(s)}|P| z\right)$ multiplied by $\mathbb{C}_{m_{1}, \alpha_{1}}^{(+) \alpha} \cdot \mathbb{C}_{\alpha, \alpha_{2}}^{(+) m_{2}}$ corresponds to one choice of the charges of the Young diagrams $\left(Y_{1}^{q_{1}}, Y_{2}^{q_{2}}\right)$ and the $\mathcal{F}^{(2)}\left(\Delta_{m_{1}}^{(s)}, \Delta_{\alpha_{1}}^{(s)}, \Delta_{\alpha_{2}}^{(s)}, \Delta_{m_{2}}^{(s)}|-P| z\right)$ multiplied by $\mathbb{C}_{m_{1}, \alpha_{1}}^{(-) \alpha} \cdot \mathbb{C}_{\alpha, \alpha_{2}}^{(-) m_{2}}$ corresponds to the Young diagrams with exchanged $\mathbb{Z}_{4}$-charges $\left(Y_{1}^{q_{2}}, Y_{2}^{q_{1}}\right)$.

So, briefly, the exchange of the $\mathbb{Z}_{4}$-charges of the Young diagrams in the gauge theory corresponds to the flip of the signs in the matrix elements with modules of the inverse Gram/Shapovalov matrix at the levels $l \in \mathbb{Z}_{\geqslant 0}+\frac{3}{4}$ on the CFT side. The same situation occurs at the level $7 / 4$.

\subsubsection{Level 1}

The basis of states at this level can be chosen in the following way

$$
\begin{aligned}
& |1\rangle_{1}=G_{-\frac{2}{3}}^{-} G_{-\frac{1}{3}}^{+}|\alpha ; 0\rangle, \\
& |2\rangle_{1}=G_{-\frac{2}{3}}^{+} G_{-\frac{1}{3}}^{-}|\alpha ; 0\rangle .
\end{aligned}
$$

We must evaluate the following quantity

$$
\sum_{i, j=1}^{2}\left\langle m_{1} ; 0\left|W_{\alpha_{1}}^{(s)}(1)\right| i\right\rangle_{1} \times\left(K_{\alpha}^{-1}(1)\right)_{i j} \times{ }_{1}\left\langle j\left|W_{\alpha_{2}}^{(s)}(z)\right| m_{2} ; 0\right\rangle .
$$

The Gram/Shapovalov matrix at this level is

$$
K_{\alpha}(1)=\frac{\Delta_{\alpha}^{(s)}}{3}\left(\begin{array}{cc}
2 \Delta_{\alpha}^{(s)}+\frac{c}{4}+1 & -\left(2 \Delta_{\alpha}^{(s)}+\frac{c}{4}-2\right) \\
-\left(2 \Delta_{\alpha}^{(s)}+\frac{c}{4}-2\right) & 2 \Delta_{\alpha}^{(s)}+\frac{c}{4}+1
\end{array}\right) .
$$

The inverse Gram/Shapovalov matrix is

$$
K_{\alpha}^{-1}(1)=\left(\begin{array}{cc}
\frac{8 \Delta_{\alpha}^{(s)}+c+4}{2 \Delta_{\alpha}^{(s)}\left(8 \Delta_{\alpha}^{(s)}+c-2\right)} & \frac{8 \Delta_{\alpha}^{(s)}+c-8}{2 \Delta_{\alpha}^{(s)}\left(8 \Delta_{\alpha}^{(s)}+c-2\right)} \\
\frac{8 \Delta_{\alpha}^{(s)}+c-8}{2 \Delta_{\alpha}^{(s)}\left(8 \Delta_{\alpha}^{(s)}+c-2\right)} & \frac{8 \Delta_{\alpha}^{(s)}+c+4}{2 \Delta_{\alpha}^{(s)}\left(8 \Delta_{\alpha}^{(s)}+c-2\right)}
\end{array}\right)
$$

After that we are ready to proceed with the calculation of matrix elements using the commutation relations (4.31) and (4.33)

$$
\begin{aligned}
& \left\langle m_{1} ; 0\left|W_{\alpha_{1}}(1)\right| 1\right\rangle_{1}=\frac{1}{2}\left(\Delta_{\alpha}^{(s)}+\Delta_{\alpha_{1}}^{(s)}-\Delta_{m_{1}}^{(s)}\right) \times C_{m_{1}, \alpha_{1}}^{\alpha}-\tilde{C}_{m_{1}, \alpha_{1}}^{\alpha}, \\
& \left\langle m_{1} ; 0\left|W_{\alpha_{1}}(1)\right| 2\right\rangle_{1}=\frac{1}{2}\left(\Delta_{\alpha}^{(s)}+\Delta_{\alpha_{1}}^{(s)}-\Delta_{m_{1}}^{(s)}\right) \times C_{m_{1}, \alpha_{1}}^{\alpha}+\tilde{C}_{m_{1}, \alpha_{1}}^{\alpha}, \\
& { }_{1}\left\langle 1\left|W_{\alpha_{2}}(z)\right| m_{2} ; 0\right\rangle=z^{\Delta_{\alpha}^{(s)}-\Delta_{\alpha_{2}}^{(s)}-\Delta_{m_{2}}^{(s)}+1} \times\left(\frac{1}{2}\left(\Delta_{\alpha}^{(s)}+\Delta_{\alpha_{2}}^{(s)}-\Delta_{m_{2}}^{(s)}\right) \times C_{\alpha, \alpha_{2}}^{m_{2}}-\tilde{C}_{\alpha, \alpha_{2}}^{m_{2}}\right), \\
& { }_{1}\left\langle 2\left|W_{\alpha_{2}}(z)\right| m_{2} ; 0\right\rangle=z^{\Delta_{\alpha}^{(s)}-\Delta_{\alpha_{2}}^{(s)}-\Delta_{m_{2}}^{(s)}+1} \times\left(\frac{1}{2}\left(\Delta_{\alpha}^{(s)}+\Delta_{\alpha_{2}}^{(s)}-\Delta_{m_{2}}^{(s)}\right) \times C_{\alpha, \alpha_{2}}^{m_{2}}+\tilde{C}_{\alpha, \alpha_{2}}^{m_{2}}\right) .
\end{aligned}
$$


Substituting the matrix elements (4.44) and the inverse Gram/Shapovalov matrix (4.43) into (4.41) and making again some algebra we obtain the expressions for the coefficients of the conformal blocks at the level 1:

$$
\begin{aligned}
& \mathcal{F}_{1}^{(1)}=\frac{\left(\Delta_{\alpha}^{(s)}+\Delta_{\alpha_{1}}^{(s)}-\Delta_{m_{1}}^{(s)}\right)\left(\Delta_{\alpha}^{(s)}+\Delta_{\alpha_{2}}^{(s)}-\Delta_{m_{2}}^{(s)}\right)}{2 \Delta_{\alpha}^{(s)}}, \\
& \mathcal{F}_{1}^{(3)}=\frac{3}{2 \Delta_{\alpha}^{(s)}\left(\Delta_{\alpha}^{(s)}+\frac{c}{8}-\frac{1}{4}\right)} .
\end{aligned}
$$

Note that there are no contributions proportional to $C_{m_{1}, \alpha_{1}}^{\alpha} \cdot \tilde{C}_{\alpha, \alpha_{2}}^{m_{2}}$ or $\tilde{C}_{m_{1}, \alpha_{1}}^{\alpha} \cdot C_{\alpha, \alpha_{2}}^{m_{2}}$. These contributions disappear from the answer due to the symmetry of the inverse Gram/Shapovalov matrix. Note that the result is in agreement with the formula (4.26).

\subsubsection{Level $7 / 4$}

Because the expressions for the conformal blocks at the level $7 / 4$ are very cumbersome, here we just write the basis of states at the level $7 / 4$

$$
\begin{aligned}
|1\rangle_{7 / 4} & =G_{-\frac{5}{3}}^{+}|\alpha ;-1\rangle, \\
|2\rangle_{7 / 4} & =G_{-\frac{5}{3}}^{-}|\alpha ; 1\rangle, \\
|3\rangle_{7 / 4} & =L_{-1} G_{-\frac{2}{3}}^{+}|\alpha ;-1\rangle, \\
|4\rangle_{7 / 4} & =L_{-1} G_{-\frac{2}{3}}^{-}|\alpha ; 1\rangle .
\end{aligned}
$$

We must evaluate the following quantity

$$
\sum_{i, j=1}^{4}\left\langle m_{1} ; 0\left|W_{\alpha_{1}}^{(s)}(1)\right| i\right\rangle_{7 / 4} \times\left(K_{\alpha}^{-1}(7 / 4)\right)_{i j} \times{ }_{7 / 4}\left\langle j\left|W_{\alpha_{2}}^{(s)}(z)\right| m_{2} ; 0\right\rangle .
$$

One can find the expression for the Gram/Shapovalov matrix and matrix elements in the Appendix C. Using the Gram/Shapovalov matrix and the matrix elements from the Appendix C one can easily obtain the expression for the conformal block. Note that there are also no contributions proportional to $\mathbb{C}_{m_{1}, \alpha_{1}}^{(+) \alpha} \mathbb{C}_{\alpha, \alpha_{2}}^{(-) m_{2}}$ or $\mathbb{C}_{m_{1}, \alpha_{1}}^{(-) \alpha} \mathbb{C}_{\alpha, \alpha_{2}}^{(+) m_{2}}$ at this level.

\subsubsection{Level 2}

The basis of states at this level can be chosen in the following way

$$
\begin{aligned}
& |1\rangle_{2}=L_{-1} G_{-\frac{2}{3}}^{-} G_{-\frac{1}{3}}^{+}|\alpha ; 0\rangle, \\
& |2\rangle_{2}=L_{-1} G_{-\frac{2}{3}}^{+} G_{-\frac{1}{3}}^{-}|\alpha ; 0\rangle, \\
& |3\rangle_{2}=G_{-\frac{5}{3}}^{-} G_{-\frac{1}{3}}^{+}|\alpha ; 0\rangle, \\
& |4\rangle_{2}=G_{-\frac{5}{3}}^{+} G_{-\frac{1}{3}}^{-}|\alpha ; 0\rangle, \\
& |5\rangle_{2}=L_{-2}|\alpha ; 0\rangle .
\end{aligned}
$$


We must evaluate the following quantity

$$
\sum_{i, j=1}^{5}\left\langle m_{1} ; 0\left|W_{\alpha_{1}}^{(s)}(1)\right| i\right\rangle_{2} \times\left(K_{\alpha}^{-1}(2)\right)_{i j} \times{ }_{2}\left\langle j\left|W_{\alpha_{2}}^{(s)}(z)\right| m_{2} ; 0\right\rangle .
$$

One can find the expression for the Gram/Shapovalov matrix and and matrix elements in the Appendix D. Using the Gram/Shapovalov matrix and the matrix elements from the Appendix D one can easily obtain the expression for the conformal block. Note that at the level 2 the contributions with $C_{m_{1}, \alpha_{1}}^{\alpha} \cdot \tilde{C}_{\alpha, \alpha_{2}}^{m_{2}}$ or $\tilde{C}_{m_{1}, \alpha_{1}}^{\alpha} \cdot C_{\alpha, \alpha_{2}}^{m_{2}}$ disappear again.

\section{Comparing the conformal blocks in the $S$ and $D$ modules of the $S_{3}$ parafermion algebra with the instanton partition function on $\mathbb{R}_{4} / \mathbb{Z}_{p}$}

Recalling that in our notations $\Delta_{P_{i}}=\Delta_{m_{i}}^{(s)}$ for $i=1,2$ because $m_{i}=\frac{Q}{2}+P_{i}$ and using the equations for the conformal block contribution and the partition function, we find, comparing the formulae (3.2) and (4.39), that at the level 3/4:

$$
\mathcal{Z}_{3 / 4}^{(4,2 \text { nd series })}=-\frac{1}{8} \mathcal{F}_{3 / 4}^{(2)}
$$

Comparing the formulas (3.4), (3.5) with (4.45), (4.46) we have at the level 1:

$$
\begin{aligned}
& \mathcal{Z}_{1}^{(4,1 \text { st series })}=\mathcal{F}_{1}^{(1)}-A_{4}, \\
& \mathcal{Z}_{1}^{(4,3 \text { rd series })}=\frac{1}{64} \mathcal{F}_{1}^{(3)} .
\end{aligned}
$$

At the level 7/4:

$$
\mathcal{Z}_{7 / 4}^{(4,2 \text { nd series })}=-\frac{1}{8}\left(\mathcal{F}_{7 / 4}^{(2)}-A_{4} \mathcal{F}_{3 / 4}^{(2)}\right)
$$

At the level 2:

$$
\begin{aligned}
& \mathcal{Z}_{2}^{(4,1 \text { st series })}=\mathcal{F}_{2}^{(1)}-A_{4} \mathcal{F}_{1}^{(1)}+\frac{1}{2} A_{4}\left(A_{4}-1\right), \\
& \mathcal{Z}_{2}^{(4,3 \text { rd series })}=\frac{1}{64}\left(\mathcal{F}_{1}^{(3)}-A_{4} \mathcal{F}_{0}^{(3)}\right)
\end{aligned}
$$

where $A_{4}$ is

$$
A_{4}=\frac{1}{2} \alpha_{1}\left(Q-\alpha_{2}\right)
$$

These checks at the particular levels lead us to the natural proposition

$$
\begin{aligned}
& \mathcal{Z}^{(4,1 \text { st series })}\left(P_{1}, \alpha_{1}, \alpha_{2}, P_{2}|P| z\right)=(1-z)^{A_{4}} \mathcal{F}^{(1)}\left(\Delta_{P_{1}}, \Delta_{\alpha_{1}}, \Delta_{\alpha_{2}}, \Delta_{P_{2}}\left|\Delta_{P}\right| z\right), \\
& \mathcal{Z}^{(4,2 \text { nd series })}\left(P_{1}, \alpha_{1}, \alpha_{2}, P_{2}|P| z\right)=-\frac{1}{2^{3}}(1-z)^{A_{4}} \mathcal{F}^{(2)}\left(\Delta_{P_{1}}, \Delta_{\alpha_{1}}, \Delta_{\alpha_{2}}, \Delta_{P_{2}}|P| z\right), \\
& \mathcal{Z}^{(4,3 \text { rd series })}\left(P_{1}, \alpha_{1}, \alpha_{2}, P_{2}|P| z\right)=\frac{1}{2^{6}}(1-z)^{A_{4}} \mathcal{F}^{(3)}\left(\Delta_{P_{1}}, \Delta_{\alpha_{1}}, \Delta_{\alpha_{2}}, \Delta_{P_{2}}\left|\Delta_{P}\right| z\right) .
\end{aligned}
$$

The formulas (5.8), (5.9) and (5.10) are the main result of our paper. 


\section{Concluding remarks and open problems}

This paper established the explicit view of relation between $S U(2)$ Nekrasov instanton partition function on $\mathbb{R}^{4} / \mathbb{Z}_{4}$ and four-point conformal blocks in $S$ and $D$ modules of the $S_{3}$ parafermion algebra. This relation can be schematically written as $\mathcal{Z}_{\text {instanton }}(z)=(1-z)^{A_{4}} \mathcal{F}_{\text {conformal block }}(z)$.

One can notice that the four-point coset conformal blocks depend on the dimensions of the primary fields in the correlation function and the parameter $P$, which denotes the intermediate parameter in the conformal block. As usual one parameterizes the conformal dimensions as $\Delta_{i}$ by the momenta $P_{i}$ as $\Delta_{i}=\frac{1}{p}\left(\frac{Q^{2}}{4}-P_{i}^{2}\right)$. Therefore the coset conformal blocks are the functions of the parameters $P_{i}^{2}$ and $P$, so $\mathcal{F}_{\text {conformal block }}=f\left(P_{i}^{2}, P \mid z\right)$. On the other hand the instanton partition function on $\mathbb{R}^{4} / \mathbb{Z}_{p}$ is a function of parameters $P_{i}, P$. We made the explicit checks for the cases of $p=2, \ldots, 7$ that the combination

$$
(1-z)^{-A_{p}} \mathcal{Z}_{\text {instanton }}^{(p)}\left(P_{i}, P \mid z\right)=\phi\left(P_{i}^{2}, P \mid z\right),
$$

where $\phi$ is some function, which depends on $P_{i}^{2}$, not on $P_{i}$ ! Thus, one can assume that the relation

$$
\mathcal{Z}_{\text {instanton }}^{(p)}\left(P_{i}, P \mid z\right)=(1-z)^{A_{p}} \mathcal{F}_{\text {conformal block }}\left(\Delta_{i}, P \mid z\right)
$$

holds for all $p\left(A_{p}=\frac{2}{p}\left(Q / 2+P_{2}\right)\left(Q / 2-P_{3}\right)\right)$.

In this paper we use the particular series in the gauge theory $\left(c_{1}(E)=0\right)$ and the particular modules $(S$ and $D)$ in the conformal field theory. As it was recently shown in the work [26], one can assume that for the coincidence in other modules of CFT we should consider other series of the Young diagrams in the gauge theory.

Also it should be mentioned that there is another formula for the instanton partition function on $\mathbb{R}^{4} / \mathbb{Z}_{p}[17,27-29]$. It can be schematically viewed as

$$
\mathcal{Z}_{\text {instanton }}^{(p)}=l_{\text {factor }} \cdot \underbrace{\mathcal{Z}_{\text {instanton }}^{(1)} \times \mathcal{Z}_{\text {instanton }}^{(1)} \times \ldots \times \mathcal{Z}_{\text {instanton }}^{(1)}}_{p},
$$

where $l_{\text {factor }}$ is the so called blow-up factor. This structure has a very clear explanation from the CFT point view [30, 31]. Factor $l_{\text {factor }}$ arises from the matrix elements of highest weight vectors of $p$ pairs of commuting Virasoro algebras, which are constructed from another algebras. The construction of two commuting Virasoro algebras using the NSR algebra and free fermion field was proposed in [32,33. Also, relations between parafermion Liouville structure constant [34,35] and Liouville structure constants

$$
C_{\text {Parafermionic Liouville }}^{(p)}=\underbrace{C_{\text {Liouville }}^{(1)} \times C_{\text {Liouville }}^{(1)} \times \ldots \times C_{\text {Liouville }}^{(1)}}_{p}
$$

in principle can be checked using the relations between $\Upsilon$-functions [31].

\section{Acknowledgments}

We thank Alexey Litvinov, Alexander Belavin, Mikhail Bershtein and Rubik Poghossian for very useful discussions.

Moral support by San'kin M. was extremely important for G. T., particularly while staying in the hospital with broken leg.

The work M.A. and G.T. was supported by 2011 Dynasty foundation grant. Also the work of G.T. was held within the framework of the Federal programs "Scientific and Scientific-Pedagogical Personnel of Innovational Russia" on 2009-2013 (state contracts No. P1339 and No. 02.740.11.5165) and was supported by cooperative CNRS-RFBR grant PICS-09-02-93064 and by Russian Ministry of Science and Technology under the Scientific Schools grant 6501.2010.2. 


\section{Appendix A. Commutation relations in the $S$-module}

Let $W_{\alpha}^{(s)}(z)$ be the highest weight state in the $S$-module, and $V_{\alpha}^{(s) \pm}(z)=G_{-1 / 3}^{ \pm} W_{\alpha}^{(s)}(z)$ be its first fractional descendants. In the work [25] one can find the OPEs for the operators $T(z)$ and $G^{ \pm}(z)$ with the vertex operators in the $S$-module

$$
\begin{aligned}
& T(u) W_{\alpha}^{(s)}(z)=\frac{\Delta_{\alpha}^{(s)}}{(u-z)^{2}} W_{\alpha}^{(s)}(z)+\frac{1}{u-z} \partial W_{\alpha}^{(s)}(z)+\cdots, \\
& T(u) V_{\alpha}^{(s) \pm}(z)=\frac{\Delta_{\alpha}^{(s)}+\frac{1}{3}}{(u-z)^{2}} V_{\alpha}^{(s) \pm}(z)+\frac{1}{u-z} \partial V_{\alpha}^{(s) \pm}(z)+\cdots, \\
& G^{ \pm}(u) W_{\alpha}^{(s)}(z)=\frac{1}{u-z} V_{\alpha}^{(s) \pm}(z)+\cdots, \\
& G^{ \pm}(u) V_{\alpha}^{(s) \pm}(z)=\frac{\lambda^{ \pm}}{2(u-z)^{\frac{4}{3}}} V_{\alpha}^{(s) \mp}(z)+\frac{1}{(u-z)^{\frac{1}{3}}}\left(\frac{\lambda^{ \pm}}{3 \Delta_{\alpha}^{(s)}+1} \partial V_{\alpha}^{(s) \mp}(z)+\tilde{V}_{\alpha}^{(s) \mp}(z)\right)+\cdots, \\
& G^{ \pm}(u) V_{\alpha}^{(s) \mp}(z)=\frac{\Delta_{\alpha}^{(s)}}{(u-z)^{\frac{5}{3}}} W_{\alpha}^{(s)}(z)+\frac{1}{(u-z)^{\frac{2}{3}}}\left(\frac{1}{2} \partial W_{\alpha}^{(s)}(z) \pm \tilde{W}_{\alpha}^{(s)}(z)\right) \cdots,
\end{aligned}
$$

where the vertex operators $\tilde{W}_{\alpha}^{(s)}(z)$ and $\tilde{V}^{(s) \pm}(z)$ correspond to the states

$$
\begin{aligned}
\left|\tilde{W}_{\alpha}^{(s)}\right\rangle & =\left(G_{-\frac{2}{3}}^{+} G_{-\frac{1}{3}}^{-}-G_{-\frac{2}{3}}^{-} G_{-\frac{1}{3}}^{+}\right)\left|W_{\alpha}^{(s)}\right\rangle, \\
\left|\tilde{V}^{(s) \pm}\right\rangle & =G_{-1}^{\mp}\left|V_{\alpha}^{(s) \mp}\right\rangle-\frac{\lambda^{\mp}}{3 h_{s}+1} L_{-1}\left|V_{\alpha}^{(s) \pm}\right\rangle .
\end{aligned}
$$

Then due to the fact that the OPEs (A.1), (A.2) and (A.3) contain only integer powers it is easy to evaluate the corresponding commutation relations

$$
\begin{aligned}
{\left[L_{m}, W_{\alpha}^{(s)}(z)\right] } & =\oint_{z} \frac{d u}{2 \pi i} u^{m+1} T(u) W_{\alpha}^{(s)}(z)=\oint_{z} \frac{d u}{2 \pi i} u^{m+1}\left(\frac{\Delta_{\alpha}^{(s)}}{(u-z)^{2}} W_{\alpha}^{(s)}(z)+\frac{1}{u-z} \partial W_{\alpha}^{(s)}(z)+\cdots\right)= \\
& =z^{m} \partial W_{\alpha}^{(s)}(z)+(m+1) \Delta_{\alpha}^{(s)} W_{\alpha}^{(s)}(z) \\
{\left[L_{m}, V_{\alpha}^{(s) \pm}(z)\right] } & =\oint_{z} \frac{d u}{2 \pi i} u^{m+1} T(u) V_{\alpha}^{(s) \pm}(z)=\oint_{z} \frac{d u}{2 \pi i} u^{m+1}\left(\frac{\Delta_{\alpha}^{(s)}+\frac{1}{3}}{(u-z)^{2}} V_{\alpha}^{(s) \pm}(z)+\frac{1}{u-z} \partial V_{\alpha}^{(s) \pm}(z)+\cdots\right)= \\
& =z^{m} \partial V_{\alpha}^{(s) \pm}(z)+(m+1)\left(\Delta_{\alpha}^{(s)}+\frac{1}{3}\right) V_{\alpha}^{(s) \pm}(z) \\
{\left[G_{r}^{ \pm}, W_{\alpha}^{(s)}(z)\right] } & =\oint_{z} \frac{d u}{2 \pi i} u^{r+\frac{1}{3}} G^{ \pm}(u) W_{\alpha}^{(s)}(z)=\oint_{z} \frac{d u}{2 \pi i} u^{r+\frac{1}{3}}\left(\frac{V_{\alpha}^{(s) \pm}(z)}{u-z}+\ldots\right)=z^{r+\frac{1}{3}} V_{\alpha}^{(s) \pm}(z) .
\end{aligned}
$$

Although due to the presence of the fractional powers in the OPEs (A.4) and (A.5) it is impossible to derive the commutation relations for these vertex operators, but it is possible to derive the generalized commutation relations in analogy with the modes of $G^{ \pm}(u)$. To do this consider the integral with $p \in \mathbb{Z}$ 
with the points $z$ and 0 inside the integration contour

$$
\begin{aligned}
\oint_{\text {incl. } z} \frac{d u}{2 \pi i} u^{r+\frac{1}{3}}(u-z)^{p+\frac{1}{3}} G^{ \pm}(u) V_{\alpha}^{(s) \pm}(z) & =\sum_{l=0}^{+\infty} C_{l}^{\left(p+\frac{1}{3}\right)} z^{l} \oint \frac{d u}{2 \pi i} u^{r+p+\frac{2}{3}-l} G^{ \pm}(u) V_{\alpha}^{(s) \pm}(z)= \\
& =\sum_{l=0}^{+\infty} C_{l}^{\left(p+\frac{1}{3}\right)} z^{l} G_{r+p-l+\frac{1}{3}}^{ \pm} V_{\alpha}^{(s) \pm}(z)=(\text { for } p=-1) \\
& =\sum_{l=0}^{+\infty} C_{l}^{\left(-\frac{2}{3}\right)} z^{l} G_{r-l-\frac{2}{3}}^{ \pm} V_{\alpha}^{(s) \pm}(z) .
\end{aligned}
$$

On the other side this integral can be divided into the integral over the point $z$, which is evaluated via the OPE (A.4), and the integral around 0 with $z$ outside this contour

$$
\begin{aligned}
\oint_{\text {incl } z} \frac{d u}{2 \pi i} u^{r+\frac{1}{3}}(u-z)^{p+\frac{1}{3}} G^{ \pm}(u) V_{\alpha}^{(s) \pm}(z)= & \oint_{z} \frac{d u}{2 \pi i} u^{r+\frac{1}{3}}(u-z)^{p+\frac{1}{3}} G^{ \pm}(u) V_{\alpha}^{(s) \pm}(z)+ \\
& +\oint_{\text {excl } z} \frac{d u}{2 \pi i} u^{r+\frac{1}{3}}(u-z)^{p+\frac{1}{3}} G^{ \pm}(u) V_{\alpha}^{(s) \pm}(z) .
\end{aligned}
$$

We make the further calculations for $p=-1$. The first integral on the right hand side can be easily evaluated using the OPE (A.4)

$$
\begin{aligned}
\oint_{z} \frac{d u}{2 \pi i} u^{r+\frac{1}{3}}(u-z)^{-\frac{2}{3}} G^{ \pm}(u) V_{\alpha}^{(s) \pm}(z)= & \oint_{z} \frac{d u}{2 \pi i} u^{r+\frac{1}{3}}\left(\frac{\lambda^{ \pm}}{2(u-z)^{2}} V_{\alpha}^{(s) \mp}(z)+\right. \\
& \left.+\frac{1}{u-z}\left(\frac{\lambda^{ \pm}}{3 \Delta_{\alpha}^{(s)}+1} \partial V_{\alpha}^{(s) \mp}(z)+\tilde{V}_{\alpha}^{(s) \mp}(z)\right)+\cdots\right)= \\
= & z^{r-\frac{2}{3}} \frac{\lambda^{ \pm}}{2}\left(r+\frac{1}{3}\right) V_{\alpha}^{(s) \mp}(z)+z^{r+\frac{1}{3}}\left(\frac{\lambda^{ \pm}}{3 h_{s}+1} \partial V_{\alpha}^{(s) \mp}(z)+\tilde{V}_{\alpha}^{(s) \mp}(z)\right) .
\end{aligned}
$$

The second integral on the right hand side must be evaluated using the abelian braiding of $G^{ \pm}$and $V_{s}^{ \pm}$, which gives the factor $e^{\frac{2 i \pi}{3}}$.

$$
\begin{aligned}
\oint_{\text {excl } z} \frac{d u}{2 \pi i} u^{r+\frac{1}{3}}(u-z)^{p+\frac{1}{3}} G^{ \pm}(u) V_{\alpha}^{(s) \pm}(z) & =e^{i \pi\left(p+\frac{1}{3}\right)} e^{\frac{2 i \pi}{3}} \oint_{\text {excl } z} \frac{d u}{2 \pi i} u^{r+\frac{1}{3}}(z-u)^{p+\frac{1}{3}} V_{\alpha}^{(s) \pm}(z) G^{ \pm}(u)= \\
& =(-1)^{p+1} \oint_{\text {excl } z} \frac{d u}{2 \pi i} u^{r+\frac{1}{3}}(z-u)^{p+\frac{1}{3}} V_{\alpha}^{(s) \pm}(z) G^{ \pm}(u)= \\
& =(-1)^{p+1} \sum_{l=0}^{+\infty} C_{l}^{\left(p+\frac{1}{3}\right)} z^{p-l+\frac{1}{3}} \oint_{\text {excl } z} \frac{d u}{2 \pi i} u^{r+l+\frac{1}{3}} V_{\alpha}^{(s) \pm}(z) G^{ \pm}(u)= \\
& =(-1)^{p+1} \sum_{l=0}^{+\infty} C_{l}^{\left(p+\frac{1}{3}\right)} z^{p-l+\frac{1}{3}} V_{\alpha}^{(s) \pm}(z) G_{r+l}^{ \pm}=(\text {for } p=-1) \\
& =\sum_{l=0}^{+\infty} C_{l}^{\left(-\frac{2}{3}\right)} z^{-l-\frac{2}{3}} V_{\alpha}^{(s) \pm}(z) G_{r+l}^{ \pm} .
\end{aligned}
$$


Summarizing (A.11), (A.13) and (A.14) we are able to write the generalized commutation relations for $p=-1$

$$
\begin{aligned}
& \sum_{l=0}^{+\infty} C_{l}^{\left(-\frac{2}{3}\right)}\left(z^{l} G_{r-l-\frac{2}{3}}^{ \pm} V_{\alpha}^{(s) \pm}(z)-z^{-l-\frac{2}{3}} V_{\alpha}^{(s) \pm}(z) G_{r+l}^{ \pm}\right)= \\
& =z^{r-\frac{2}{3}} \frac{\lambda^{ \pm}}{2}\left(r+\frac{1}{3}\right) V_{\alpha}^{(s) \mp}(z)+z^{r+\frac{1}{3}}\left(\frac{\lambda^{ \pm}}{3 h_{s}+1} \partial V_{\alpha}^{(s) \mp}(z)+\tilde{V}_{\alpha}^{(s) \mp}(z)\right)
\end{aligned}
$$

where the descendant $V_{\alpha}^{(s) \pm}(z)$ is given by the formula (A.7).

Then with the opposite $\mathbb{Z}_{3}$-charges

$$
\begin{aligned}
\oint_{\text {incl } z} \frac{d u}{2 \pi i} u^{r+\frac{1}{3}}(u-z)^{p+\frac{2}{3}} G^{ \pm}(u) V_{\alpha}^{(s) \mp}(z) & =\sum_{l=0}^{+\infty} C_{l}^{\left(p+\frac{2}{3}\right)} z^{l} \oint_{i n c l z} \frac{d u}{2 \pi i} u^{r+p+1-l} G^{ \pm}(u) V_{\alpha}^{(s) \mp}(z)= \\
& =\sum_{l=0}^{+\infty} C_{l}^{\left(p+\frac{2}{3}\right)} z^{l} G_{r+p-l+\frac{2}{3}}^{ \pm} V_{\alpha}^{(s) \mp}(z)=(\text { for } p=-1) \\
& =\sum_{l=0}^{+\infty} C_{l}^{\left(-\frac{1}{3}\right)} z^{l} G_{r-l-\frac{1}{3}}^{ \pm} V_{\alpha}^{(s) \mp}(z) .
\end{aligned}
$$

On the other side this integral can be divided into the integral over the point $z$, which is evaluated via the OPE (A.5), and the integral around 0 with $z$ outside this contour

$$
\begin{aligned}
\oint_{\text {incl } z} \frac{d u}{2 \pi i} u^{r+\frac{1}{3}}(u-z)^{p+\frac{2}{3}} G^{ \pm}(u) V_{\alpha}^{(s) \mp}(z)= & \oint_{z} \frac{d u}{2 \pi i} u^{r+\frac{1}{3}}(u-z)^{p+\frac{2}{3}} G^{ \pm}(u) V_{\alpha}^{(s) \mp}(z)+ \\
& +\oint_{\text {excl } z} \frac{d u}{2 \pi i} u^{r+\frac{1}{3}}(u-z)^{p+\frac{2}{3}} G^{ \pm}(u) V_{\alpha}^{(s) \mp}(z) .
\end{aligned}
$$

To evaluate the first term on the right hand side of (A.17) we use the OPE (4.3) (we again set $p=-1$ )

$$
\begin{aligned}
\oint_{z} \frac{d u}{2 \pi i} u^{r+\frac{1}{3}}(u-z)^{-\frac{1}{3}} G^{ \pm}(u) V_{\alpha}^{(s) \mp}(z)= & \oint_{z} \frac{d u}{2 \pi i} u^{r+\frac{1}{3}}\left(\frac{\Delta_{\alpha}^{(s)}}{(u-z)^{2}} W_{\alpha}^{(s)}(z)+\right. \\
& \left.+\frac{1}{u-z}\left(\partial W_{\alpha}^{(s)}(z) \pm \tilde{W}_{\alpha}^{(s)}(z)\right)+\cdots\right)= \\
= & z^{r-\frac{2}{3}}\left(r+\frac{1}{3}\right) \Delta_{\alpha}^{(s)} W_{\alpha}^{(s)}(z)+z^{r+\frac{1}{3}}\left(\frac{1}{2} \partial W_{\alpha}^{(s)}(z) \pm \tilde{W}_{\alpha}^{(s)}(z)\right)
\end{aligned}
$$


The abelian braiding of the fields $G^{ \pm}(u)$ and $V^{(s) \mp}(z)$ gives the factor $e^{-\frac{2 i \pi}{3}}$

$$
\begin{aligned}
\oint_{\text {excl } z} \frac{d u}{2 \pi i} u^{r+\frac{1}{3}}(u-z)^{p+\frac{2}{3}} G^{ \pm}(u) V_{\alpha}^{(s) \mp}(z) & =e^{i \pi\left(p+\frac{2}{3}\right)} e^{-\frac{2 i \pi}{3}} \oint_{\text {excl } z} \frac{d u}{2 \pi i} u^{r+\frac{1}{3}}(z-u)^{p+\frac{2}{3}} V_{\alpha}^{(s) \mp}(z) G^{ \pm}(u)= \\
& =(-1)^{p} \oint_{\text {excl } z} \frac{d u}{2 \pi i} u^{r+\frac{1}{3}}(z-u)^{p+\frac{2}{3}} V_{\alpha}^{(s) \mp}(z) G^{ \pm}(u)= \\
& =(-1)^{p} \sum_{l=0}^{+\infty} C_{l}^{\left(p+\frac{2}{3}\right)} z^{p-l+\frac{2}{3}} \oint_{\text {excl } z} \frac{d u}{2 \pi i} u^{r+l+\frac{1}{3}} V_{\alpha}^{(s) \mp}(z) G^{ \pm}(u)= \\
& =(-1)^{p} \sum_{l=0}^{+\infty} C_{l}^{\left(p+\frac{2}{3}\right)} z^{p-l+\frac{2}{3}} V_{\alpha}^{(s) \mp}(z) G_{r+l}^{ \pm}=(\text {for } p=-1) \\
& =-\sum_{l=0}^{+\infty} C_{l}^{\left(-\frac{1}{3}\right)} z^{-l-\frac{1}{3}} V_{\alpha}^{(s) \mp}(z) G_{r+l}^{ \pm} .
\end{aligned}
$$

After that summarizing (A.16), (A.18) and (A.19) we are able to write the generalized commutation relations

$$
\begin{aligned}
& \sum_{l=0}^{+\infty} C_{l}^{\left(-\frac{1}{3}\right)}\left(z^{l} G_{r-l-\frac{1}{3}}^{ \pm} V_{\alpha}^{(s) \mp}(z)+z^{-l-\frac{1}{3}} V_{\alpha}^{(s) \mp}(z) G_{r+l}^{ \pm}\right)= \\
& =z^{r-\frac{2}{3}}\left(r+\frac{1}{3}\right) \Delta_{\alpha}^{(s)} W_{\alpha}^{(s)}(z)+z^{r+\frac{1}{3}}\left(\frac{1}{2} \partial W_{\alpha}^{(s)}(z) \pm \tilde{W}_{\alpha}^{(s)}(z)\right)
\end{aligned}
$$

where the descendant $\tilde{W}_{\alpha}^{(s)}(z)$ is given by the formula (A.6).

\section{Appendix B. Commutation relations in the $D$-module}

Let $W_{\alpha}^{(d) \pm}(z)$ be the vertex operators of the primary states in the $D$-module. To find the commutation relations we need the OPEs [25] of the fields $W_{\alpha}^{(d) \pm}(z)$ and their first conformal descendants $V_{\alpha}^{(d)( \pm)}$

$$
\begin{aligned}
T(u) W_{\alpha}^{(d) \pm}(z) & =\frac{\Delta_{\alpha}^{(d)}}{(u-z)^{2}} W_{\alpha}^{(d) \pm}(z)+\frac{1}{u-z} \partial W_{\alpha}^{(d) \pm}(z)+\cdots, \\
T(u) V_{\alpha}^{(d)( \pm)}(z)= & \frac{\Delta_{\alpha}^{(d)}+\frac{2}{3}}{(u-z)^{2}} V_{\alpha}^{(d)( \pm)}(z)+\frac{1}{u-z} \partial V_{\alpha}^{(d)( \pm)}(z)+\cdots, \\
G^{ \pm}(u) W_{\alpha}^{(d) \pm}(z)= & \frac{\Lambda^{ \pm}}{(u-z)^{\frac{4}{3}}} W_{\alpha}^{(d) \pm}(z)+\frac{1}{(u-z)^{\frac{1}{3}}}\left(\frac{2 \Lambda^{ \pm}}{3 \Delta_{\alpha}^{(d)}} \partial W_{\alpha}^{(d) \pm}(z)+\tilde{W}_{\alpha}^{(d) \pm}(z)\right)+\cdots, \\
G^{ \pm}(u) W_{\alpha}^{(d) \mp}(z)= & \frac{1}{2(u-z)^{\frac{2}{3}}}\left(V_{\alpha}^{(d)(+)}(z) \pm V_{\alpha}^{(d)(+)}(z)\right)+\cdots, \\
G^{ \pm}(u) V_{\alpha}^{(d)(+)}(z)= & \frac{\Delta_{\alpha}^{(d)}+\frac{c}{12}+\lambda^{ \pm} \Lambda^{\mp}}{(u-z)^{2}} W_{\alpha}^{(d) \pm}(z)+ \\
& +\frac{1}{u-z}\left(\frac{\Delta_{\alpha}^{(d)}+\frac{c}{12}+\lambda^{ \pm} \Lambda^{\mp}}{3 \Delta_{\alpha}^{(s)}} \partial W_{\alpha}^{(d) \pm}(z)-\left(\Lambda^{ \pm}-\frac{1}{2} \lambda^{ \pm}\right) \tilde{W}_{\alpha}^{(d) \pm}(z)\right)+\cdots
\end{aligned}
$$




$$
\begin{aligned}
G^{ \pm}(u) V_{\alpha}^{(d)(-)}(z)= & \mp \frac{\Delta_{\alpha}^{(d)}+\frac{c}{12}-\lambda^{ \pm} \Lambda^{\mp}}{(u-z)^{2}} W_{\alpha}^{(d) \pm}(z)+ \\
& +\frac{1}{u-z}\left(\mp \frac{\Delta_{\alpha}^{(d)}+\frac{c}{12}-\lambda^{ \pm} \Lambda^{\mp}}{3 \Delta_{\alpha}^{(s)}} \partial W_{\alpha}^{(d) \pm}(z) \pm\left(\Lambda^{ \pm}+\frac{1}{2} \lambda^{ \pm}\right) \tilde{W}_{\alpha}^{(d) \pm}(z)\right)+\cdots,
\end{aligned}
$$

where the vertex operators $V_{\alpha}^{(d)( \pm)}(z)$ and $\tilde{W}_{\alpha}^{(d) \pm}(z)$ correspond to the states

$$
\begin{aligned}
& \left|V_{\alpha}^{(d)( \pm)}\right\rangle=G_{-\frac{2}{3}}^{+}\left|W_{\alpha}^{(d)-}\right\rangle \pm G_{-\frac{2}{3}}^{-}\left|W_{\alpha}^{(d)+}\right\rangle, \\
& \left|\tilde{W}_{\alpha}^{(d) \pm}\right\rangle=G_{-1}^{\mp}\left|W_{\alpha}^{(d) \mp}\right\rangle-\frac{2 \Lambda^{\mp}}{3 \Delta_{\alpha}^{(d)}} L_{-1}\left|W_{\alpha}^{(d) \pm}\right\rangle .
\end{aligned}
$$

Due to the fact that the OPEs (B.1), (B.2), (B.5) and (B.6) contain only integer powers of $u-z$, we are able to write the commutation relations

$$
\begin{aligned}
& {\left[L_{m}, W_{\alpha}^{(d) \pm}(z)\right]=\oint_{z} \frac{d u}{2 \pi i} u^{m+1} T(u) W_{\alpha}^{(d) \pm}(z)=\oint_{z} \frac{d u}{2 \pi i} u^{m+1}\left(\frac{\Delta_{\alpha}^{(d)}}{(u-z)^{2}} W_{\alpha}^{(d) \pm}(z)+\frac{1}{u-z} \partial W_{\alpha}^{(d) \pm}(z)+\cdots\right)=} \\
& =z^{m} \partial W_{\alpha}^{(d) \pm}(z)+(m+1) \Delta_{\alpha}^{(d)} W_{\alpha}^{(d) \pm}(z) \\
& {\left[L_{m}, V_{\alpha}^{(d)( \pm)}(z)\right]=\oint_{z} \frac{d u}{2 \pi i} u^{m+1} T(u) V_{\alpha}^{(d)( \pm)}(z)=\oint_{z} \frac{d u}{2 \pi i} u^{m+1}\left(\frac{\Delta_{\alpha}^{(d)}+\frac{2}{3}}{(u-z)^{2}} V_{\alpha}^{(s)( \pm)}(z)+\frac{1}{u-z} \partial V_{\alpha}^{(d)( \pm)}(z)+. .\right)=} \\
& =z^{m} \partial V_{\alpha}^{(d)( \pm)}(z)+(m+1)\left(\Delta_{\alpha}^{(d)}+\frac{2}{3}\right) V_{\alpha}^{(d)( \pm)}(z), \\
& {\left[G_{r}^{ \pm}, V_{\alpha}^{(d)+}(z)\right]=\oint_{z} \frac{d u}{2 \pi i} u^{r+\frac{1}{3}} G^{ \pm}(u) V_{\alpha}^{(d)+}(z)=\oint_{z} \frac{d u}{2 \pi i} u^{r+\frac{1}{3}}\left(\frac{\Delta_{\alpha}^{(d)}+\frac{c}{12}+\lambda^{ \pm} \Lambda^{\mp}}{(u-z)^{2}} W_{\alpha}^{(d) \pm}(z)+\right.} \\
& \left.+\frac{1}{u-z}\left(\frac{\Delta_{\alpha}^{(d)}+\frac{c}{12}+\lambda^{ \pm} \Lambda^{\mp}}{3 \Delta_{\alpha}^{(s)}} \partial W_{\alpha}^{(d) \pm}(z)-\left(\Lambda^{ \pm}-\frac{1}{2} \lambda^{ \pm}\right) \tilde{W}_{\alpha}^{(d) \pm}(z)\right)+\cdots\right)= \\
& =z^{r-\frac{2}{3}}\left(r+\frac{1}{3}\right)\left(\Delta_{\alpha}^{(d)}+\frac{c}{12}+\lambda^{ \pm} \Lambda^{\mp}\right) W_{\alpha}^{(d) \pm}(z)+z^{r+\frac{1}{3}} \frac{\Delta_{\alpha}^{(d)}+\frac{c}{12}+\lambda^{ \pm} \Lambda^{\mp}}{3 \Delta_{\alpha}^{(d)}} \partial W_{\alpha}^{(d) \pm}(z)- \\
& -z^{r+\frac{1}{3}}\left(\Lambda^{ \pm}-\frac{1}{2} \lambda^{ \pm}\right) \tilde{W}_{\alpha}^{(d) \pm}(z) \\
& {\left[G_{r}^{ \pm}, V_{\alpha}^{(d)-}(z)\right]=\oint_{z} \frac{d u}{2 \pi i} u^{r+\frac{1}{3}} G^{ \pm}(u) V_{\alpha}^{(d)-}(z)=\oint_{z} \frac{d u}{2 \pi i} u^{r+\frac{1}{3}}\left(\mp \frac{\Delta_{\alpha}^{(d)}+\frac{c}{12}-\lambda^{ \pm} \Lambda^{\mp}}{(u-z)^{2}} W_{\alpha}^{(d) \pm}(z)+\right.} \\
& \left.+\frac{1}{u-z}\left(\mp \frac{\Delta_{\alpha}^{(d)}+\frac{c}{12}-\lambda^{ \pm} \Lambda^{\mp}}{3 \Delta_{\alpha}^{(s)}} \partial W_{\alpha}^{(d) \pm}(z) \pm\left(\Lambda^{ \pm}+\frac{1}{2} \lambda^{ \pm}\right) \tilde{W}_{\alpha}^{(d) \pm}(z)\right)+\cdots\right)= \\
& \mp z^{r-\frac{2}{3}}\left(r+\frac{1}{3}\right)\left(\Delta_{\alpha}^{(d)}+\frac{c}{12}-\lambda^{ \pm} \Lambda^{\mp}\right) W_{\alpha}^{(d) \pm}(z) \mp z^{r+\frac{1}{3}} \frac{\Delta_{\alpha}^{(d)}+\frac{c}{12}-\lambda^{ \pm} \Lambda^{\mp}}{3 \Delta_{\alpha}^{(d)}} \partial W_{\alpha}^{(d) \pm}(z) \pm \\
& \pm z^{r+\frac{1}{3}}\left(\Lambda^{ \pm}+\frac{1}{2} \lambda^{ \pm}\right) \tilde{W}_{\alpha}^{(d) \pm}(z)
\end{aligned}
$$


where the descendant $\tilde{W}_{\alpha}^{(d) \pm}(z)$ is given by the formula (B.8).

Analogously to the case of the $S$-module

$$
\begin{aligned}
\oint_{\text {incl } z} \frac{d u}{2 \pi i} u^{r+\frac{1}{3}}(u-z)^{p+\frac{1}{3}} G^{ \pm}(u) W_{\alpha}^{(d) \pm}(z)= & \oint_{z} \frac{d u}{2 \pi i} u^{r+\frac{1}{3}}(u-z)^{p+\frac{1}{3}} G^{ \pm}(u) W_{\alpha}^{(d) \pm}(z)+ \\
& +\oint_{\text {excl } z} \frac{d u}{2 \pi i} u^{r+\frac{1}{3}}(u-z)^{p+\frac{1}{3}} G^{ \pm}(u) W_{\alpha}^{(d) \pm}(z) .
\end{aligned}
$$

The contours encircling the origin can be easily evaluated using the abelian braiding of $G^{ \pm}(u)$ with $W_{\alpha}^{(d) \pm}(z)$, which gives in this case the factor $e^{\frac{2 i \pi}{3}}$

$$
\begin{aligned}
& \oint_{\text {incl } z} \frac{d u}{2 \pi i} u^{r+\frac{1}{3}}(u-z)^{p+\frac{1}{3}} G^{ \pm}(u) W_{\alpha}^{(d) \pm}(z)=\sum_{l=0}^{+\infty} C_{l}^{\left(p+\frac{1}{3}\right)} z^{l} G_{r+p-l+\frac{1}{3}}^{ \pm} W_{\alpha}^{(d) \pm}(z), \\
& \oint_{\text {excl } z} \frac{d u}{2 \pi i} u^{r+\frac{1}{3}}(u-z)^{p+\frac{1}{3}} G^{ \pm}(u) W_{\alpha}^{(d) \pm}(z)=(-1)^{p+1} \sum_{l=0}^{+\infty} C_{l}^{\left(p+\frac{1}{3}\right)} z^{p-l+\frac{1}{3}} W_{\alpha}^{(d) \pm}(z) G_{r+l}^{ \pm} .
\end{aligned}
$$

The term with the contour encircling the point $z$ is calculated using the OPE $(\underline{\mathrm{B} .3})$ in the $D$-module (we again set $p=-1$ )

$$
\begin{aligned}
\oint_{z} \frac{d u}{2 \pi i} u^{r+\frac{1}{3}}(u-z)^{-\frac{2}{3}} G^{ \pm}(u) W_{\alpha}^{(d) \pm}(z)= & \oint_{z} \frac{d u}{2 \pi i} u^{r+\frac{1}{3}}\left(\frac{\Lambda^{ \pm}}{(u-z)^{2}} W_{\alpha}^{(d) \pm}(z)+\right. \\
& \left.+\frac{1}{u-z}\left(\frac{2 \Lambda^{ \pm}}{3 \Delta_{\alpha}^{(d)}} \partial W_{\alpha}^{(d) \pm}(z)+\tilde{W}_{\alpha}^{(d) \pm}(z)\right)+\cdots\right)= \\
= & z^{r-\frac{2}{3}}\left(r+\frac{1}{3}\right) \Lambda^{ \pm} W_{\alpha}^{(d) \mp}(z)+z^{r+\frac{1}{3}}\left(\frac{2 \Lambda^{ \pm}}{3 \Delta_{\alpha}^{(d)}} \partial W_{\alpha}^{(d) \mp}(z)+\tilde{W}_{\alpha}^{(d) \mp}(z)\right) .
\end{aligned}
$$

Then summarizing the derived equations (B.14), (B.15) and (B.16) we have

$$
\begin{aligned}
\sum_{l=0}^{+\infty} C_{l}^{\left(-\frac{2}{3}\right)}\left(z^{l} G_{r-l-\frac{2}{3}}^{ \pm}\right. & \left.W_{\alpha}^{(d) \pm}(z)-z^{-l-\frac{2}{3}} W_{\alpha}^{(d) \pm}(z) G_{r+l}^{ \pm}\right)= \\
= & z^{r-\frac{2}{3}}\left(r+\frac{1}{3}\right) \Lambda^{ \pm} W_{\alpha}^{(d) \mp}(z)+z^{r+\frac{1}{3}}\left(\frac{2 \Lambda^{ \pm}}{3 \Delta_{\alpha}^{(d)}} \partial W_{\alpha}^{(d) \mp}(z)+\tilde{W}_{\alpha}^{(d) \mp}(z)\right) .
\end{aligned}
$$

The same thing with the opposite signs of $\mathbb{Z}_{3}$-charge

$$
\begin{aligned}
\oint_{\text {incl } z} \frac{d u}{2 \pi i} u^{r+\frac{1}{3}}(u-z)^{p+\frac{2}{3}} G^{ \pm}(u) W_{\alpha}^{(d) \mp}(z)= & \oint_{z} \frac{d u}{2 \pi i} u^{r+\frac{1}{3}}(u-z)^{p+\frac{2}{3}} G^{ \pm}(u) W_{\alpha}^{(d) \mp}(z)+ \\
& +\oint_{\text {excl } z} \frac{d u}{2 \pi i} u^{r+\frac{1}{3}}(u-z)^{p+\frac{2}{3}} G^{ \pm}(u) W_{\alpha}^{(d) \mp}(z) .
\end{aligned}
$$

The contours encircling the origin can be easily evaluated using the abelian braiding of $G^{ \pm}(u)$ with $W_{\alpha}^{(d) \mp}(z)$, which gives in this case the factor $e^{-\frac{2 i \pi}{3}}$

$$
\begin{aligned}
& \oint_{\text {incl } z} \frac{d u}{2 \pi i} u^{r+\frac{1}{3}}(u-z)^{p+\frac{2}{3}} G^{ \pm}(u) W_{d}^{\mp}(z)=\sum_{l=0}^{+\infty} C_{l}^{\left(p+\frac{2}{3}\right)} z^{l} G_{r+p-l+\frac{2}{3}}^{ \pm} W_{d}^{ \pm}(z), \\
& \oint_{\text {excl } z} \frac{d u}{2 \pi i} u^{r+\frac{1}{3}}(u-z)^{p+\frac{2}{3}} G^{ \pm}(u) W_{d}^{\mp}(z)=(-1)^{p} \sum_{l=0}^{+\infty} C_{l}^{\left(p+\frac{2}{3}\right)} z^{p-l+\frac{2}{3}} W_{d}^{\mp}(z) G_{r+l}^{ \pm} .
\end{aligned}
$$


The term with the contour encircling the point $z$ is calculated using the OPE (B.4) in the $D$-module (we again set $p=-1$ )

$$
\begin{aligned}
\oint_{z} \frac{d u}{2 \pi i} u^{r+\frac{1}{3}}(u-z)^{-\frac{1}{3}} G^{ \pm}(u) W_{\alpha}^{(d) \mp}(z) & =\oint_{z} \frac{d u}{2 \pi i} u^{r+\frac{1}{3}}\left(\frac{1}{2(u-z)}\left(V_{\alpha}^{(d)(+)}(z) \pm V_{\alpha}^{(d)(+)}(z)\right)+\cdots\right)= \\
& =\frac{1}{2} z^{r+\frac{1}{3}}\left(V_{\alpha}^{(d)(+)}(z) \pm V_{\alpha}^{(d)(-)}(z)\right) .
\end{aligned}
$$

After the above operations, summarizing $(\overline{B .19}),(\bar{B} .20)$ and $(\overline{B .21})$, we are able to write the generalized commutation relations

$$
\sum_{l=0}^{+\infty} C_{l}^{\left(-\frac{1}{3}\right)}\left(z^{l} G_{r-l-\frac{1}{3}}^{ \pm} W_{\alpha}^{(d) \mp}(z)+z^{-l-\frac{1}{3}} W_{\alpha}^{(d) \mp}(z) G_{r+l}^{ \pm}\right)=\frac{1}{2} z^{r+\frac{1}{3}}\left(V_{\alpha}^{(d)(+)}(z) \pm V_{\alpha}^{(d)(-)}(z)\right),
$$

where the descendants $V_{\alpha}^{(d)( \pm)}(z)$ is given by the formula (B.7).

\section{Appendix C. Gram/Shapovalov matrix and matrix elements at the level $7 / 4$}

The Gram/Shapovalov matrix at the level 1

$$
\left(\begin{array}{cccc}
-\left(\frac{2}{3} \Delta_{\alpha}^{(d)}+\frac{5 c}{9}\right) & \frac{4}{3} \sqrt{\frac{c-8}{6}} \sqrt{\frac{c}{24}-\Delta_{\alpha}^{(d)}} & -2\left(\Delta_{\alpha}^{(d)}+\frac{c}{12}\right) & 2 \sqrt{\frac{c-8}{6}} \sqrt{\frac{c}{24}-\Delta_{\alpha}^{(d)}} \\
\frac{4}{3} \sqrt{\frac{c-8}{6}} \sqrt{\frac{c}{24}-\Delta_{\alpha}^{(d)}} & -\left(\frac{2}{3} \Delta_{\alpha}^{(d)}+\frac{5 c}{9}\right) & 2 \sqrt{\frac{c-8}{6}} \sqrt{\frac{c}{24}-\Delta_{\alpha}^{(d)}} & -2\left(\Delta_{\alpha}^{(d)}+\frac{c}{12}\right) \\
-2\left(\Delta_{\alpha}^{(d)}+\frac{c}{12}\right) & 2 \sqrt{\frac{c-8}{6}} \sqrt{\frac{c}{24}-\Delta_{\alpha}^{(d)}} & -2\left(\Delta_{\alpha}^{(d)}+\frac{2}{3}\right)\left(\Delta_{\alpha}^{(d)}+\frac{c}{12}\right) & 2\left(\Delta_{\alpha}^{(d)}+\frac{2}{3}\right) \sqrt{\frac{c-8}{6}} \sqrt{\frac{c}{24}-\Delta_{\alpha}^{(d)}} \\
2 \sqrt{\frac{c-8}{6}} \sqrt{\frac{c}{24}-\Delta_{\alpha}^{(d)}} & -2\left(\Delta_{\alpha}^{(d)}+\frac{c}{12}\right) & 2\left(\Delta_{\alpha}^{(d)}+\frac{2}{3}\right) \sqrt{\frac{c-8}{6}} \sqrt{\frac{c}{24}-\Delta_{\alpha}^{(d)}} & -2\left(\Delta_{\alpha}^{(d)}+\frac{2}{3}\right)\left(\Delta_{\alpha}^{(d)}+\frac{c}{12}\right)
\end{array}\right) .
$$

The corresponding matrix elements

$$
\begin{aligned}
& \left\langle m_{1} ; 0\left|W_{\alpha_{1}}(1)\right| 1\right\rangle_{7 / 4}=-\left(\mathbb{C}_{m_{1}, \alpha_{1}}^{(+) \alpha}+\mathbb{C}_{m_{1}, \alpha_{1}}^{(-) \alpha}\right), \\
& \left\langle m_{1} ; 0\left|W_{\alpha_{1}}(1)\right| 2\right\rangle_{7 / 4}=-\left(\mathbb{C}_{m_{1}, \alpha_{1}}^{(+) \alpha}-\mathbb{C}_{m_{1}, \alpha_{1}}^{(-) \alpha}\right), \\
& \left\langle m_{1} ; 0\left|W_{\alpha_{1}}(1)\right| 3\right\rangle_{7 / 4}=\left(\Delta_{m_{1}}^{(s)}-\Delta_{\alpha_{1}}^{(s)}-\Delta_{\alpha}^{(d)}-\frac{2}{3}\right) \times\left(\mathbb{C}_{m_{1}, \alpha_{1}}^{(+) \alpha}+\mathbb{C}_{m_{1}, \alpha_{1}}^{(-) \alpha}\right), \\
& \left\langle m_{1} ; 0\left|W_{\alpha_{1}}(1)\right| 4\right\rangle_{7 / 4}=\left(\Delta_{m_{1}}^{(s)}-\Delta_{\alpha_{1}}^{(s)}-\Delta_{\alpha}^{(d)}-\frac{2}{3}\right) \times\left(\mathbb{C}_{m_{1}, \alpha_{1}}^{(+) \alpha}-\mathbb{C}_{m_{1}, \alpha_{1}}^{(-) \alpha}\right), \\
& { }_{7 / 4}\left\langle 1\left|W_{\alpha_{2}}(z)\right| m_{2} ; 0\right\rangle=-z^{\Delta_{\alpha}^{(d)}+\frac{5}{3}-\Delta_{\alpha_{2}}^{(s)}-\Delta_{m_{2}}^{(s)}} \times\left(\mathbb{C}_{\alpha, \alpha_{2}}^{(+) m_{2}}+\mathbb{C}_{\alpha, \alpha_{2}}^{(-) m_{2}}\right), \\
& { }_{7 / 4}\left\langle 2\left|W_{\alpha_{2}}(z)\right| m_{2} ; 0\right\rangle=-z^{\Delta_{\alpha}^{(d)}+\frac{5}{3}-\Delta_{\alpha_{2}}^{(s)}-\Delta_{m_{2}}^{(s)}} \times\left(\mathbb{C}_{\alpha, \alpha_{2}}^{(+) m_{2}}-\mathbb{C}_{\alpha, \alpha_{2}}^{(-) m_{2}}\right), \\
& { }_{7 / 4}\left\langle 3\left|W_{\alpha_{2}}(z)\right| m_{2} ; 0\right\rangle=-\left(\Delta_{\alpha}^{(d)}+\frac{2}{3}+\Delta_{\alpha_{2}}^{(s)}-\Delta_{m_{2}}^{(s)}\right) z^{\Delta_{\alpha}^{(d)}+\frac{5}{3}-\Delta_{\alpha_{2}}^{(s)}-\Delta_{m_{2}}^{(s)}} \times\left(\mathbb{C}_{\alpha, \alpha_{2}}^{(+) m_{2}}+\mathbb{C}_{\alpha, \alpha_{2}}^{(-) m_{2}}\right), \\
& { }_{7 / 4}\left\langle 4\left|W_{\alpha_{2}}(z)\right| m_{2} ; 0\right\rangle=-\left(\Delta_{\alpha}^{(d)}+\frac{2}{3}+\Delta_{\alpha_{2}}^{(s)}-\Delta_{m_{2}}^{(s)}\right) z^{\Delta_{\alpha}^{(d)}+\frac{5}{3}-\Delta_{\alpha_{2}}^{(s)}-\Delta_{m_{2}}^{(s)}} \times\left(\mathbb{C}_{\alpha, \alpha_{2}}^{(+) m_{2}}-\mathbb{C}_{\alpha, \alpha_{2}}^{(-) m_{2}}\right) .
\end{aligned}
$$




\section{Appendix D. Gram/Shapovalov matrix and matrix elements at the level 2}

The corresponding Gram/Shapovalov matrix is
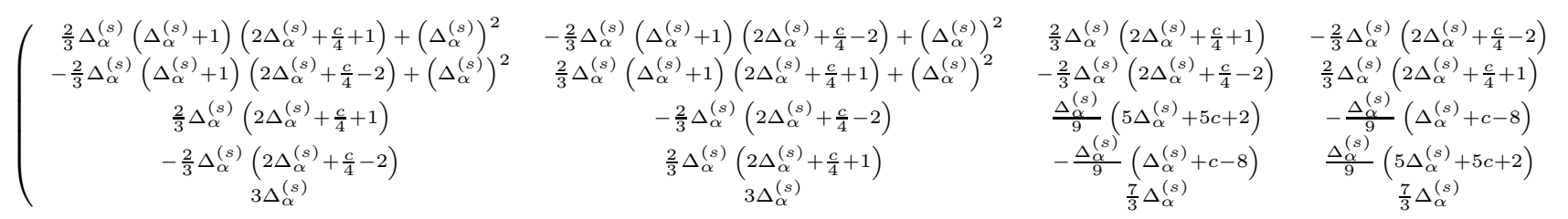

$3 \Delta_{\alpha}^{(s)}$ $3 \Delta_{\alpha}^{(s)}$ $\frac{7}{3} \Delta_{\alpha}^{(s)}$ $\frac{7}{3} \Delta_{\alpha}^{(s)}$ $4\left(\Delta_{\alpha}^{(s)}+\frac{c}{8}\right)$

The matrix elements have the corresponding form

$$
\begin{aligned}
& \left\langle m_{1} ; 0\left|W_{\alpha_{1}}(1)\right| 1\right\rangle_{2}=\frac{1}{2}\left(\Delta_{m_{1}}^{(s)}-\Delta_{\alpha_{1}}^{(s)}-\Delta_{\alpha}^{(s)}\right)\left(\Delta_{m_{1}}^{(s)}-\Delta_{\alpha_{1}}^{(s)}-\Delta_{\alpha}^{(s)}-1\right) \times C_{m_{1}, \alpha_{1}}^{\alpha}+ \\
& +\left(\Delta_{m_{1}}^{(s)}-\Delta_{\alpha_{1}}^{(s)}-\Delta_{\alpha}^{(s)}-1\right) \times \tilde{C}_{m_{1}, \alpha_{1}}^{\alpha}, \\
& \left\langle m_{1} ; 0\left|W_{\alpha_{1}}(1)\right| 2\right\rangle_{2}=\frac{1}{2}\left(\Delta_{m_{1}}^{(s)}-\Delta_{\alpha_{1}}^{(s)}-\Delta_{\alpha}^{(s)}\right)\left(\Delta_{m_{1}}^{(s)}-\Delta_{\alpha_{1}}^{(s)}-\Delta_{\alpha}^{(s)}-1\right) \times C_{m_{1}, \alpha_{1}}^{\alpha}- \\
& -\left(\Delta_{m_{1}}^{(s)}-\Delta_{\alpha_{1}}^{(s)}-\Delta_{\alpha}^{(s)}-1\right) \times \tilde{C}_{m_{1}, \alpha_{1}}^{\alpha}, \\
& \left\langle m_{1} ; 0\left|W_{\alpha_{1}}(1)\right| 3\right\rangle_{2}=-\frac{1}{2}\left(\Delta_{m_{1}}^{(s)}-\Delta_{\alpha_{1}}^{(s)}-\Delta_{\alpha}^{(s)}\right) \times C_{m_{1}, \alpha_{1}}^{\alpha}-\tilde{C}_{m_{1}, \alpha_{1}}^{\alpha}, \\
& \left\langle m_{1} ; 0\left|W_{\alpha_{1}}(1)\right| 4\right\rangle_{2}=-\frac{1}{2}\left(\Delta_{m_{1}}^{(s)}-\Delta_{\alpha_{1}}^{(s)}-\Delta_{\alpha}^{(s)}\right) \times C_{m_{1}, \alpha_{1}}^{\alpha}-\tilde{C}_{m_{1}, \alpha_{1}}^{\alpha}, \\
& \left\langle m_{1} ; 0\left|W_{\alpha_{1}}(1)\right| 5\right\rangle_{2}=\left(2 \Delta_{\alpha_{1}}^{(s)}+\Delta_{\alpha}^{(s)}-\Delta_{m_{1}}^{(s)}\right) \times C_{m_{1}, \alpha_{1}}^{\alpha}, \\
& { }_{2}\left\langle 1\left|W_{\alpha_{2}}(z)\right| m_{2} ; 0\right\rangle=z^{\Delta_{\alpha}^{(s)}-\Delta_{\alpha_{2}}^{(s)}-\Delta_{m_{2}}^{(s)}+2}\left(\frac{1}{2}\left(\Delta_{\alpha}^{(s)}+1+\Delta_{\alpha_{2}}^{(s)}-\Delta_{m_{2}}^{(s)}\right)\left(\Delta_{\alpha}^{(s)}+\Delta_{\alpha_{2}}^{(s)}-\Delta_{m_{2}}^{(s)}\right) \times C_{\alpha, \alpha_{2}}^{m_{2}}-\right. \\
& \left.-\left(\Delta_{\alpha}^{(s)}+1+\Delta_{\alpha_{2}}^{(s)}-\Delta_{m_{2}}^{(s)}\right) \times \tilde{C}_{\alpha, \alpha_{2}}^{m_{2}}\right), \\
& { }_{2}\left\langle 2\left|W_{\alpha_{2}}(z)\right| m_{2} ; 0\right\rangle=z^{\Delta_{\alpha}^{(s)}-\Delta_{\alpha_{2}}^{(s)}-\Delta_{m_{2}}^{(s)}+2}\left(\frac{1}{2}\left(\Delta_{\alpha}^{(s)}+1+\Delta_{\alpha_{2}}^{(s)}-\Delta_{m_{2}}^{(s)}\right)\left(\Delta_{\alpha}^{(s)}+\Delta_{\alpha_{2}}^{(s)}-\Delta_{m_{2}}^{(s)}\right) \times C_{\alpha, \alpha_{2}}^{m_{2}}+\right. \\
& \left.+\left(\Delta_{\alpha}^{(s)}+1+\Delta_{\alpha_{2}}^{(s)}-\Delta_{m_{2}}^{(s)}\right) \times \tilde{C}_{\alpha, \alpha_{2}}^{m_{2}}\right), \\
& { }_{2}\left\langle 3\left|W_{\alpha_{2}}(z)\right| m_{2} ; 0\right\rangle=z^{\Delta_{\alpha}^{(s)}-\Delta_{\alpha_{2}}^{(s)}-\Delta_{m_{2}}^{(s)}+2}\left(\frac{1}{2}\left(\Delta_{\alpha}^{(s)}+\Delta_{\alpha_{2}}^{(s)}-\Delta_{m_{2}}^{(s)}\right) \times C_{\alpha, \alpha_{2}}^{m_{2}}-\tilde{C}_{\alpha, \alpha_{2}}^{m_{2}}\right), \\
& { }_{2}\left\langle 4\left|W_{\alpha_{2}}(z)\right| m_{2} ; 0\right\rangle=z^{\Delta_{\alpha}^{(s)}-\Delta_{\alpha_{2}}^{(s)}-\Delta_{m_{2}}^{(s)}+2}\left(\frac{1}{2}\left(\Delta_{\alpha}^{(s)}+\Delta_{\alpha_{2}}^{(s)}-\Delta_{m_{2}}^{(s)}\right) \times C_{\alpha, \alpha_{2}}^{m_{2}}+\tilde{C}_{\alpha, \alpha_{2}}^{m_{2}}\right), \\
& { }_{2}\left\langle 5\left|W_{\alpha_{2}}(z)\right| m_{2} ; 0\right\rangle=z^{\Delta_{\alpha}^{(s)}-\Delta_{\alpha_{2}}^{(s)}-\Delta_{m_{2}}^{(s)}+2}\left(2 \Delta_{\alpha_{2}}^{(s)}+\Delta_{\alpha}^{(s)}-\Delta_{m_{2}}^{(s)}\right) \times C_{\alpha, \alpha_{2}}^{m_{2}} .
\end{aligned}
$$

\section{References}

[1] L. F. Alday, D. Gaiotto, and Y. Tachikawa, Liouville correlation functions from four-dimensional gauge theories, Lett. Math. Phys. 91 (2010) 167-197, [arXiv:0906.3219].

[2] V. A. Fateev and A. V. Litvinov, On AGT conjecture, JHEP 02 (2010) 014, [arXiv:0912.0504]. 
[3] L. Hadasz, Z. Jaskolski, and P. Suchanek, Proving the AGT relation for $N_{f}=0,1,2$ antifundamentals, JHEP 06 (2010) 046, [arXiv:1004.1841].

[4] R. Poghossian, Recursion relations in CFT and N=2 SYM theory, JHEP 12 (2009) 038, [arXiv:0909.3412].

[5] A. Mironov and A. Morozov, The power of Nekrasov functions, Phys. Lett. B680 (2009) 188-194, [arXiv:0908.2190].

[6] A. Mironov and A. Morozov, Proving AGT relations in the large-c limit, Phys. Lett. B682 (2009) 118-124, [arXiv:0909.3531].

[7] R. Dijkgraaf and C. Vafa, Toda theories, matrix models, topological strings, and N=2 gauge systems, arXiv:0909.2453.

[8] M. C. Cheng, R. Dijkgraaf, and C. Vafa, Non-perturbative topological strings and conformal blocks, JHEP 1109 (2011) 022, [arXiv:1010.4573].

[9] A. Mironov, A. Morozov, and S. Shakirov, A direct proof of AGT conjecture at beta $=1$, JHEP 1102 (2011) 067, [arXiv:1012.3137].

[10] A. Mironov, A. Morozov, and S. Shakirov, Towards a proof of AGT conjecture by methods of matrix models, arXiv:1011.5629.

[11] A. Braverman, B. Feigin, M. Finkelberg, and L. Rybnikov, A finite analog of the AGT relation I: finite $W$-algebras and quasimaps' spaces, Commun. Math. Phys. (2011) [arXiv:1008.3655].

[12] H. Awata, B. Feigin, A. Hoshino, M. Kanai, J. Shiraishi, and S. Yanagida, Notes on Ding-Iohara algebra and AGT conjecture, arXiv:1106.4088.

[13] V. Belavin and B. Feigin, Super Liouville conformal blocks from N=2 SU(2) quiver gauge theories, JHEP 1107 (2011) 079, [arXiv:1105.5800].

[14] T. Nishioka and Y. Tachikawa, Central charges of para-Liouville and Toda theories from M-5-branes, Phys.Rev. D84 (2011) 046009, [arXiv:1106.1172].

[15] G. Bonelli, K. Maruyoshi, and A. Tanzini, Instantons on ALE spaces and Super Liouville Conformal Field Theories, JHEP 1108 (2011) 056, [arXiv:1106.2505].

[16] A. Belavin, V. Belavin, and M. Bershtein, Instantons and 2d Superconformal field theory, JHEP 1109 (2011) 117, [arXiv:1106.4001].

[17] G. Bonelli, K. Maruyoshi, and A. Tanzini, Gauge Theories on ALE Space and Super Liouville Correlation Functions, arXiv:1107.4609.

[18] N. Wyllard, Coset conformal blocks and N=2 gauge theories, arXiv:1109.4264.

[19] V. A. Fateev and A. B. Zamolodchikov, Representations of the algebra of parafermion currents of spin 4/3 in two-dimensional conformal field theory. Minimal models and the tricritical Potts $Z(3)$ model, Theor. Math. Phys. 71 (1987) 451-462.

[20] F. Fucito, J. F. Morales, and R. Poghossian, Multi instanton calculus on ALE spaces, Nucl.Phys. B703 (2004) 518-536, hep-th/0406243. 
[21] F. Fucito, J. F. Morales, and R. Poghossian, Instanton on toric singularities and black hole countings, JHEP 12 (2006) 073, hep-th/0610154.

[22] R. Flume and R. Poghossian, An algorithm for the microscopic evaluation of the coefficients of the Seiberg-Witten prepotential, Int. J. Mod. Phys. A18 (2003) 2541, hep-th/0208176.

[23] N. A. Nekrasov, Seiberg-Witten Prepotential From Instanton Counting, Adv. Theor. Math. Phys. 7 (2004) 831-864, hep-th/0206161.

[24] G. W. Moore, N. Nekrasov, and S. Shatashvili, Integrating over Higgs branches, Commun. Math. Phys. 209 (2000) 97-121, hep-th/9712241.

[25] P. C. Argyres and S. H. H. Tye, Tree scattering amplitudes of the spin 4/3 fractional superstring. 1. The Untwisted sectors, Phys. Rev. D49 (1994) 5326-5348, hep-th/9310131.

[26] Y. Ito, Ramond sector of super Liouville theory from instantons on an ALE space, arXiv:1110.2176.

[27] U. Bruzzo, R. Poghossian, and A. Tanzini, Poincare polynomial of moduli spaces of framed sheaves on (stacky) Hirzebruch surfaces, Commun. Math. Phys. 304 (2011) 395-409, arXiv:0909.1458.

[28] U. Bruzzo, F. Fucito, J. F. Morales, and A. Tanzini, Multi-instanton calculus and equivariant cohomology, JHEP 05 (2003) 054, hep-th/0211108.

[29] T. Sasaki, O(-2) blow-up formula via instanton calculus on $C^{* * 2 / Z(2)-~ h a t ~ a n d ~ W e i l ~ c o n j e c t u r e, ~}$ hep-th/0603162.

[30] V. A. Alba, V. A. Fateev, A. V. Litvinov, and G. M. Tarnopolsky, On combinatorial expansion of the conformal blocks arising from AGT conjecture, Lett. Math. Phys. 98 (2011) 33-64, [arXiv:1012.1312].

[31] A. A. Belavin, M. A. Bershtein, B. L. Feigin, A. V. Litvinov, and G. M. Tarnopolsky, Instanton moduli spaces and bases in coset conformal field theory, arXiv:1111.2803.

[32] M. Lashkevich, Superconformal 2-D minimal models and an unusual coset construction, Mod. Phys. Lett. A8 (1993) 851-860, hep-th/9301093.

[33] C. Crnkovic, R. Paunov, G. Sotkov, and M. Stanishkov, Fusions of conformal models, Nucl.Phys. B336 (1990) 637.

[34] M. A. Bershtein, V. A. Fateev, and A. V. Litvinov, Parafermionic polynomials, Selberg integrals and three-point correlation function in parafermionic Liouville field theory, Nucl. Phys. B847 (2011) 413-459, [arXiv:1011.4090].

[35] R. G. Pogosian, Operator algebra in two-dimensional conformal quantum field theory containing spin 4/3 parafermionic conserved currents., Int. J. Mod. Phys. A6 (1991) 2005-2023. 